Supplementary Material for

\title{
Steric vs. Hydrogen Bonding Control of Atropisomerization: Preparation of Either Diastereomer of Configurationally Stable Allocolchicinoids
}

\author{
Andrei V. Vorogushin, William D. Wulff and Hans-Jürgen Hansen \\ Department of Chemistry \\ Michigan State University \\ East Lansing, MI 48824 and \\ Institute of Organic Chemistry \\ University of Zurich, Winterthurerstrasse 190 \\ CH-8057, Zürich, Switzerland
}

\section{Contents}

1) General Considerations 2

2) Determination of the Diastereomeric Ratio for Compounds 2, 4, 7-12.

General Procedure.

3) 8,11-Dihydroxy-1,2,3,7-tetramethoxy-10-propyl-5,6-dihydro-5H-

dibenzo[a,c]cycloheptenes (4).

4) Thermal Epimerization of Hydroquinones 4.6

5) $\quad(\mathrm{a} R, 7 S ; \mathrm{a} S, 7 R)-8$-Acetoxy-11-hydroxy-1,2,3,7-tetramethoxy-10-propyl-5,6-

dihydro-5H-dibenzo[a,c]cycloheptene (5,I) and $(\mathrm{a} R, 7 R$; aS,7S)-11-Acetoxy-8- 
hydroxy-1,2,3,7-tetramethoxy-10-propyl-5,6-dihydro-5Hdibenzo[a,c]cycloheptene $(\mathbf{6}, \mathbf{I I})$.

6) 8-Acyloxy-1,2,3,7,11-pentamethoxy-9-propyl-5,6-dihydro-5Hdibenzo[a,c]cycloheptenes. $\mathrm{R}^{2}=\mathrm{COCH}_{3}(7), \mathrm{COCMe}_{3}(\mathbf{8})$.

7) 1,2,3,7,8,11-Hexamethoxy-9-propyl-5,6-dihydro-5H-dibenzo[a,c]cycloheptenes $(\mathbf{9})$.

8) 1,2,3,7,11-Pentamethoxy-8-trialkylsiloxy-9-propyl-5,6-dihydro-5Hdibenzo[a,c]cycloheptenes. PG = TMS (10), TBDMS (11), TIPS (12); $\mathrm{R}=\mathrm{Me}, \mathrm{Et}, i$-Pr. $\mathrm{R}^{1}=\mathrm{Me}, \mathrm{Et}, i$-Pr, $t$-Bu. General Procedure.

9) $\left(\mathrm{aR}^{*}, 7 \mathrm{~S}^{*}\right)$-7,11-dialkoxy-8-hydroxy-1,2,3-trimethoxy-9-propyl-5,6dihydro-5H-dibenzo[a,c]cycloheptenes (2,I). General Procedure.

10) Thermal Epimerization of Phenols 2 and Their Protected Derivatives 7-12. $\mathrm{R}=\mathrm{Me}, \mathrm{Et}, i-\mathrm{Pr} ; \mathrm{R}^{1}=\mathrm{Me}, \mathrm{Et}, i-\mathrm{Pr}, t-\mathrm{Bu} ; \mathrm{R}^{2}=\mathrm{H}, \mathrm{Me}, \mathrm{COCH}_{3}$, $\mathrm{COCMe}_{3}, \mathrm{TBS}, \mathrm{TBDMS}, \mathrm{TIPS}$. General Procedure.

\section{General Considerations:}

Routine NMR spectra were recorded on $300 \mathrm{MHz}, 400 \mathrm{MHz}, 500 \mathrm{MHz}$ and 600 $\mathrm{MHz}$ instruments in $\mathrm{CDCl}_{3}$ (internal standard tetramethylsilane, $\delta_{\mathrm{H}}, \delta_{\mathrm{C}}=0.00 \mathrm{ppm}$ ), benzene- $\mathrm{d}_{6}\left(\delta_{\mathrm{H}}=7.15 \mathrm{ppm}, \delta_{\mathrm{C}}=128.0 \mathrm{ppm}\right)$, acetone- $\mathrm{d}_{6}\left(\delta_{\mathrm{H}}=2.04 \mathrm{ppm}, \delta_{\mathrm{C}}=29.8 \mathrm{ppm}\right)$, DMSO- $_{6}\left(\delta_{\mathrm{H}}=2.49 \mathrm{ppm}, \delta_{\mathrm{C}}=39.5 \mathrm{ppm}\right)$. Proton signal assignments in the ${ }^{1} \mathrm{H}-\mathrm{NMR}$ spectra, which where not obvious from the chemical shift and multiplicity data, were determined using standard COSY experiments. Carbon signal multiplicities in the ${ }^{13} \mathrm{C}$ 
NMR spectra were determined from standard DEPT experiments. Mass spectral analyses (low and high resolution) were performed at both Michigan State University and University of Zürich Mass Spectrometry Facilities. Anhydrous Ether and THF were distilled under nitrogen from sodium benzophenone ketyl. Anhydrous benzene and toluene were distilled under nitrogen from sodium. Anhydrous methylene chloride was distilled under nitrogen from $\mathrm{CaH}_{2}$. Unless otherwise mentioned, all other reagents were purchased from commercial sources and were used without further purification. Yields in Table 1 refer to isolated yields (after column chromatography) of compounds estimated to be more than $95 \%$ pure as determined by ${ }^{1} \mathrm{H}$ NMR or combustion analysis. Material recovery in Tables 1 and 2 refer to the mixture of diastereomers I and II obtained after column chromatography. Diastereomeric ratios (dr) in Tables 1 and 2 were determined from the crude mixture by ${ }^{1} \mathrm{H}-\mathrm{NMR}$ integration according to the following procedure.

\section{Determination of the Diastereomeric Ratio for Compounds 2, 4, 7-12.}

\section{General Procedure.}
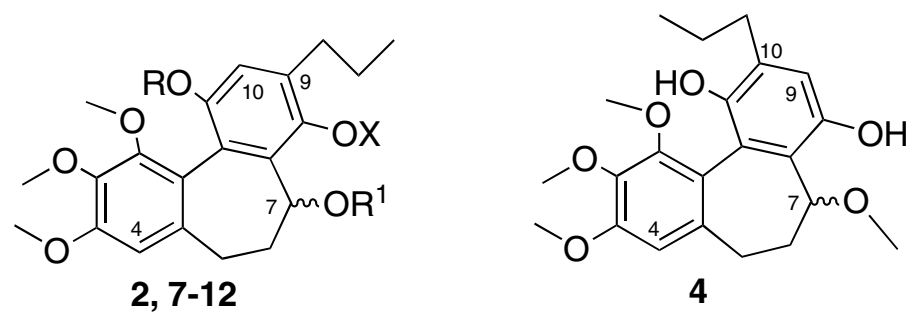

The diastereomeric ratios (dr) for $\mathbf{2 , 4 , 7 - 1 2}$ were determined from the crude mixture of diastereomers by integration of their ${ }^{1} \mathrm{H}-\mathrm{NMR}$ signals. ${ }^{1} \mathrm{H}-\mathrm{NMR}$ spectra were obtained from the solutions containing at least $30 \mathrm{mg}$ of the mixture in $0.6 \mathrm{~mL}$ of $\mathrm{CDCl}_{3}$ with at least 16 scans. Baseline correction was performed before the integration. Usually 
singlet signals of $\mathrm{C} 4-\mathrm{H}, \mathrm{C} 9-\mathrm{H}, \mathrm{C} 10-\mathrm{H}$ and $\mathrm{OCH}_{3}$ were integrated and the data was averaged where possible.

\section{8,11-Dihydroxy-1,2,3,7-tetramethoxy-10-propyl-5,6-dihydro-5H- dibenzo[a,c]cycloheptenes (4).}

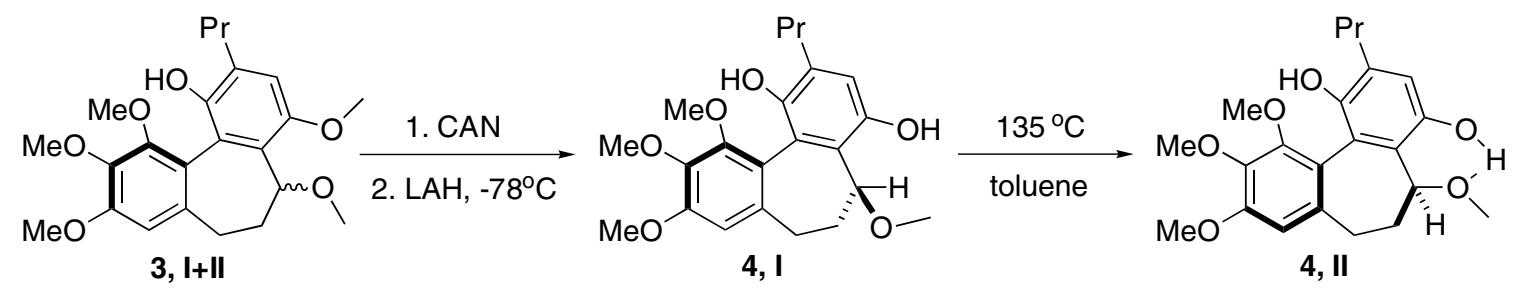

To a solution of phenol $3\left(\mathrm{R}, \mathrm{R}^{1}=\mathrm{Me}\right)^{1}(0.53 \mathrm{~g}, 1.31 \mathrm{mmol}), 3: 1$ mixture of $\left(\mathrm{a} R^{*}, 7 S^{*}\right)$-I and $\left(\mathrm{a} S^{*}, 7 S^{*}\right)$-II diastereomers, in $5 \mathrm{~mL}$ of ether was added an aqueous solution of CAN (1.436 g, $2.62 \mathrm{mmol})$ in $2.7 \mathrm{~mL}$ of water with stirring. The mixture turned red almost instantly. After 5 minutes it was diluted with water, the organic layer was separated and the aqueous layer was extracted once with ether. The etheral layers were united and dried over $\mathrm{MgSO}_{4}$. The solvent was removed in vacuo, the red residue was thoroughly dried under high vacuum and then dissolved in $25 \mathrm{~mL}$ of absolute ether. The solution was cooled to $-78{ }^{\circ} \mathrm{C}$ under Ar and a $1 \mathrm{M}$ solution of $\mathrm{LiAlH}_{4}(2.12 \mathrm{~mL}, 2.12$ mmol) in THF was added to it dropwise. The mixture was stirred at $-78{ }^{\circ} \mathrm{C}$ for 1 hour, and then cooling bath was removed and the mixture was allowed to reach room temperature. The mixture was carefully quenched under Ar by the dropwise addition of $0.5 \mathrm{~mL}$ of water followed by $4 \mathrm{~mL}$ of $10 \% \mathrm{HCl}$ solution. The organic layer was separated and the aqueous layer was extracted twice with ether. The organic layers were 
united and dried over $\mathrm{MgSO}_{4}$. The residue obtained after the solvent removal was checked by ${ }^{1} \mathrm{H}-\mathrm{NMR}$ and found to contain $\left(\mathrm{a} R^{*}, 7 S^{*}\right)$ diastereomer $\mathbf{I}$ of the target hydroquinone $\mathbf{4}$ along with oligomeric products and trace amounts of the intermediate quinone. The signals of the opposite $\left(\mathrm{a} S^{*}, 7 S^{*}\right)$ diastereomer II were not observed. The crude product was purified by column chromatography (silica gel, $30 \%$ EtOAc in hexane) to give 4,I $(0.376 \mathrm{~g}, 74 \%)$. The opposite diastereomer 4,II can be obtained selectively by thermal epimerization in toluene at $135{ }^{\circ} \mathrm{C}$ for 24 hours according to the described below procedure.

$\left(\mathrm{a} R^{*}, 7 S^{*}\right)$ diastereomer 4,I: Yellowish crystals. M.p. $=155.5-156.5^{\circ} \mathrm{C}$.

${ }^{1} \mathrm{H}-\mathrm{NMR}\left(500 \mathrm{MHz}, \mathrm{CDCl}_{3}\right) \delta 0.98(\mathrm{t}, 3 \mathrm{H}, \mathrm{J}=7.4 \mathrm{~Hz}), 1.60-1.69(\mathrm{~m}, 2 \mathrm{H}), 2.26(\mathrm{td}, 1 \mathrm{H}, \mathrm{J}$ $=13.8,5.5 \mathrm{~Hz}), 2.35-2.41(\mathrm{~m}, 2 \mathrm{H}), 2.47(\mathrm{td}, 1 \mathrm{H}, \mathrm{J}=13.1,6.5 \mathrm{~Hz}), 2.61-2.70(\mathrm{~m}, 2 \mathrm{H})$, $2.93(\mathrm{~s}, 3 \mathrm{H}), 3.72(\mathrm{~s}, 3 \mathrm{H}), 3.88(\mathrm{~s}, 3 \mathrm{H}), 3.89(\mathrm{~s}, 3 \mathrm{H}), 4.81(\mathrm{~s}, 1 \mathrm{H}), 5.03(\mathrm{~d}, 1 \mathrm{H}, \mathrm{J}=6.5$ $\mathrm{Hz}), 6.59(\mathrm{~s}, 1 \mathrm{H}), 6.63(\mathrm{~s}, 1 \mathrm{H}, \mathrm{OH}), 6.70(\mathrm{~s}, 1 \mathrm{H}) .{ }^{13} \mathrm{C}-\mathrm{NMR}\left(75 \mathrm{MHz}, \mathrm{CDCl}_{3}\right) \delta$ 14.0, $23.2,31.0,32.8,39.7,55.7,56.1,61.2,62.0,74.1,108.6,116.1,122.8,122.9,124.9$, 132.6, 137.5, 140.2, 145.5, 147.0, 149.1, 152.3; IR (mix. of diastereomers) $\left(\mathrm{KBr}, \mathrm{cm}^{-1}\right)$ 3388 (vs, OH), 2936 (m), 1603 (m), 1423 (s), 1113 (s); mass spectrum (EI) m/z (\% rel. intensity) $388 \mathrm{M}^{+}$(42), $356 \mathrm{M}^{+}-\mathrm{MeOH}$ (100), $341 \mathrm{M}^{+}$- MeOH-Me (11), 327 (21), 281 (6); HRMS (EI) calcd for $\mathrm{C}_{22} \mathrm{H}_{28} \mathrm{O}_{6} 388.1886$, found 388.1881. Anal (mix of diastereomers) calcd for $\mathrm{C}_{22} \mathrm{H}_{28} \mathrm{O}_{6}$ : C, 68.02; H, 7.27. Found C, 67.92; H, 7.43.

$\left(\mathrm{a} S^{*}, 7 S^{*}\right)$ diastereomer 4,II: Yellowish oil.

${ }^{1} \mathrm{H}-\mathrm{NMR}\left(500 \mathrm{MHz}, \mathrm{CDCl}_{3}\right) \delta 0.98(\mathrm{t}, 3 \mathrm{H}, \mathrm{J}=7.4 \mathrm{~Hz}), 1.60-1.72(\mathrm{~m}, 2 \mathrm{H}), 2.08-2.15(\mathrm{~m}$, 1H), 2.27-2.41 (m, 2H), $2.44(\mathrm{dd}, 1 \mathrm{H}, \mathrm{J}=11.4,5.2 \mathrm{~Hz}), 2.66(\mathrm{t}, 2 \mathrm{H}, \mathrm{J}=7.6 \mathrm{~Hz}), 3.40(\mathrm{~s}$,

(1) A. V. Vorogushin, W. D. Wulff, H.-J. Hansen, J. Am. Chem. Soc. 2002, 124, 6512. 
3H), 3.69 (s, 3H), 3.909 (s, 3H), $3.912(\mathrm{~s}, 3 \mathrm{H}), 4.07(\mathrm{dd}, 1 \mathrm{H}, \mathrm{J}=11.4,6.2 \mathrm{~Hz}), 6.45(\mathrm{~s}$, $1 \mathrm{H}, \mathrm{OH}), 6.64(\mathrm{~s}, 1 \mathrm{H}), 6.72(\mathrm{~s}, 1 \mathrm{H}), 8.52(\mathrm{~s}, 1 \mathrm{H}, \mathrm{OH}) ;{ }^{13} \mathrm{C}-\mathrm{NMR}\left(75 \mathrm{MHz}, \mathrm{CDCl}_{3}\right)$ $\delta 14.0,23.1,30.4,32.6,37.0,56.0,58.2,61.3,61.9,83.2,109.0,117.8,118.0,121.2$, 122.2, 132.4, 136.4, 140.7, 143.9, 148.1, 149.3, 152.9; IR (mix. of diastereomers) (KBr, $\left.\mathrm{cm}^{-1}\right) 3388(\mathrm{vs}, \mathrm{OH}), 2936(\mathrm{~m}), 1603(\mathrm{~m}), 1423(\mathrm{~s}), 1113(\mathrm{~s})$; mass spectrum (EI) $\mathrm{m} / \mathrm{z}(\%$ rel. intensity) $388 \mathrm{M}^{+}$(77), $356 \mathrm{M}^{+}-\mathrm{MeOH}$ (100), $341 \mathrm{M}^{+}-\mathrm{MeOH}$ - Me (15), 327 (27), 313 (19), 281 (9); HRMS (EI) calcd for $\mathrm{C}_{22} \mathrm{H}_{28} \mathrm{O}_{6} 388.1886$, found 388.1886. Anal (mix of diastereomers) calcd for $\mathrm{C}_{22} \mathrm{H}_{28} \mathrm{O}_{6}$ : C, 68.02; H, 7.27. Found C, 67.92; H, 7.43.

\section{Thermal Epimerization of Hydroquinones 4.}

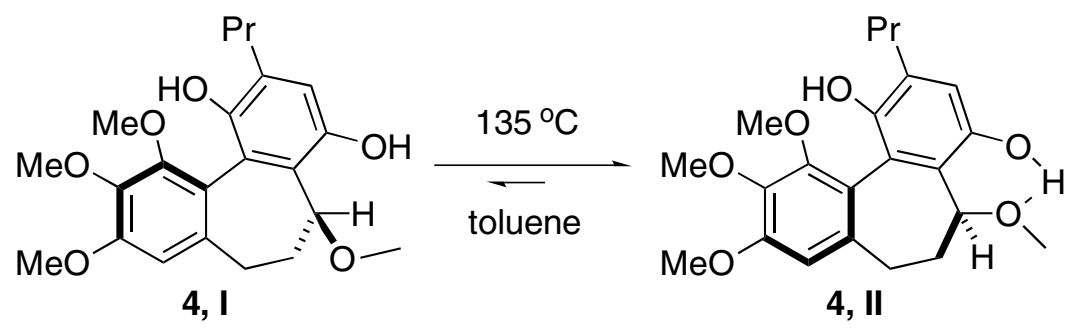

Separate solutions of individual diastereomers $\mathbf{4 , I}$ and $\mathbf{4 , I I}$ in dry toluene (10 $\mathrm{mg} / \mathrm{mL}$ ) were placed in Schlenk flasks, deoxygenated by the freeze-pump-thaw method ($196{ }^{\circ} \mathrm{C} / 25^{\circ} \mathrm{C}, 3$ cycles), then the flasks were back-filled with Ar at room temperature, sealed and placed in an oil heating bath side by side. The solutions were stirred at $135^{\circ} \mathrm{C}$ for 24 hours. TLC and HPLC analysis of the mixtures showed that the same diastereomeric ratio has been achieved in both tubes. Then the solutions were mixed together, the toluene was removed in vacuo and the diastereomeric ratio (I : I I) was found by ${ }^{1} \mathrm{H}-\mathrm{NMR}$ to be $3: 97$. After that the mixture was purified chromatographically 
on silica gel, diastereomers I + II were collected and average material recovery was determined $(92 \%)$.

$(\mathrm{a} R, 7 S ; \mathrm{a} S, 7 R)$-8-Acetoxy-11-hydroxy-1,2,3,7-tetramethoxy-10-propyl-5,6-dihydro$5 H$-dibenzo[a,c]cycloheptene $(5, \mathrm{I})$ and $(\mathrm{a} R, 7 R ; \mathrm{a} S, 7 S)$-11-Acetoxy-8-hydroxy1,2,3,7-tetramethoxy-10-propyl-5,6-dihydro-5H-dibenzo[a,c]cycloheptene (6,II).

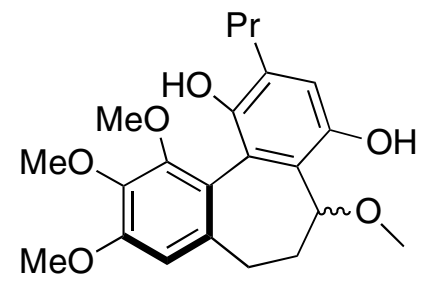

4, I. $\left(a^{\star}, 7 S^{\star}\right)$

4, II. $\left(a S^{*}, 7 S^{\star}\right)$

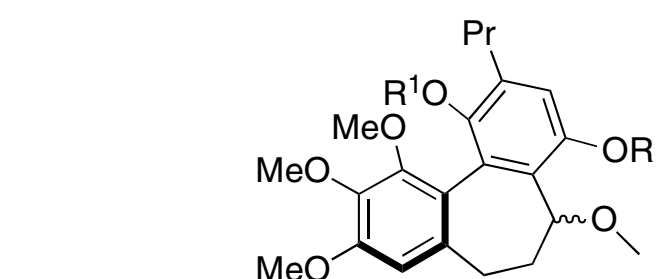

5, I. R = Ac, $\mathrm{R}^{1}=\mathrm{H} ; 87 \%$

6, II. $R=H, R^{1}=A c, 70 \%$

To a stirred solution of the selected diastereomer (I or II) of 4 (1 eq.) in dry $\mathrm{CH}_{2} \mathrm{Cl}_{2}(\sim 0.2 \mathrm{M})$ under nitrogen was added $\mathrm{Ac}_{2} \mathrm{O}(3 \mathrm{eq})$ and this was followed by the dropwise addition of pyridine ( 3 eq.). The mixture was stirred at room temperature and monitored by TLC for the disappearance of the starting material (1 hour $40 \mathrm{~min}$. for isomer I, 36 hours for isomer II). Then the mixture was quenched with water, the product was extracted with ether, organic layer was washed once with $10 \% \mathrm{HCl}$ and once with water and then dried over $\mathrm{MgSO}_{4}$. After the removal of the solvent, the residue was purified by column chromatography (silica gel, $20 \%$ EtOAc in hexane). Spectral data and yields for the products are given below:

$\left(\mathrm{a} R^{*}, 7 S^{*}\right)-\mathbf{5}$,I: Yield $87 \%$. Yellowish oil. 
${ }^{1} \mathrm{H}-\mathrm{NMR}\left(600 \mathrm{MHz}, \mathrm{CDCl}_{3}\right) \delta 0.99(\mathrm{t}, 3 \mathrm{H}, \mathrm{J}=7.3 \mathrm{~Hz}), 1.63-1.74(\mathrm{~m}, 2 \mathrm{H}), 2.21(\mathrm{td}, 1 \mathrm{H}, \mathrm{J}$ $=14.0,5.6 \mathrm{~Hz}), 2.32(\mathrm{~s}, 3 \mathrm{H}), 2.32-2.41(\mathrm{~m}, 2 \mathrm{H}), 2.47(\mathrm{td}, 1 \mathrm{H}, \mathrm{J}=13.4,6.4 \mathrm{~Hz}), 2.66-2.76$ (m, 2H), $2.88(\mathrm{~s}, 3 \mathrm{H}), 3.75(\mathrm{~s}, 3 \mathrm{H}), 3.885(\mathrm{~s}, 3 \mathrm{H}), 3.892(\mathrm{~s}, 3 \mathrm{H}), 4.54(\mathrm{~d}, 1 \mathrm{H}, \mathrm{J}=6.4 \mathrm{~Hz})$, $6.63(\mathrm{~s}, 1 \mathrm{H}), 6.84(\mathrm{~s}, 1 \mathrm{H}), 6.96\left(\mathrm{~s}, 1 \mathrm{H}, \mathrm{OH}\right.$, exchange with $\left.\mathrm{D}_{2} \mathrm{O}\right) ;{ }^{13} \mathrm{C}-\mathrm{NMR}(75 \mathrm{MHz}$, $\left.\mathrm{CDCl}_{3}\right) \delta 14.0,20.7,22.8,30.8,32.7,40.0,55.7,56.5,61.1,62.0,75.3,108.7,122.1$, $122.3,124.1,128.7,132.9,137.4,140.4,142.2,149.2,149.8,152.6,169.9$; IR (KBr, $\mathrm{cm}^{-}$ 1) $3375(\mathrm{~s}, \mathrm{OH}), 2936(\mathrm{~s}), 1762(\mathrm{~s}, \mathrm{CO}), 1603(\mathrm{~m}), 1455(\mathrm{~s}), 1208(\mathrm{~s})$; mass spectrum (EI) $m / z$ (\% rel. intensity) $430 \mathrm{M}^{+}$(44), $388 \mathrm{M}^{+}-\mathrm{CH}_{2} \mathrm{CO}(16), 356 \mathrm{M}^{+}-\mathrm{CH}_{2} \mathrm{CO}-\mathrm{MeOH}$ (66), 327 (10), 84 (84), 49 (100); HRMS (EI) calcd for $\mathrm{C}_{24} \mathrm{H}_{30} \mathrm{O}_{7}$ 430.1992, found 430.1992. Anal calcd for $\mathrm{C}_{24} \mathrm{H}_{30} \mathrm{O}_{7}$ : C, 66.96; H, 7.02. Found C, 66.90; H, 6.98.

$\left(\mathrm{a} S^{*}, 7 S^{*}\right)-\mathbf{6}$,II. Yield $70 \%$. Yellowish oil or amorphous solid.

${ }^{1} \mathrm{H}-\mathrm{NMR}\left(500 \mathrm{MHz}, \mathrm{CDCl}_{3}\right) \delta 0.96(\mathrm{t}, 3 \mathrm{H}, \mathrm{J}=7.4 \mathrm{~Hz}), 1.58-1.69(\mathrm{~m}, 2 \mathrm{H}), 1.89-2.00$ (br.s, 3H), 2.01-2.08 (m, 1H), 2.29-2.42 (m, 3H), 2.43-2.49 (m, 2H), 3.43 (s, 3H), 3.593.70 (br.s, 3H), 3.88 (s, 3H), 3.89 (s, 3H), $4.12(\mathrm{dd}, 1 \mathrm{H}, \mathrm{J}=11.4,5.6 \mathrm{~Hz}), 6.54(\mathrm{~s}, 1 \mathrm{H})$, $6.75(\mathrm{~s}, 1 \mathrm{H}), 8.84(\mathrm{~s}, 1 \mathrm{H}, \mathrm{OH}) ;{ }^{13} \mathrm{C}-\mathrm{NMR}\left(125 \mathrm{MHz}, \mathrm{CDCl}_{3}\right) \delta$ 14.0, 20.5, 22.8, 29.6, $32.3,36.7,55.8,58.4,60.9,60.9,83.3,107.2,117.1,118.7,120.1,127.7,134.7,135.8$ 139.3, 140.3, 150.8, 152.3, 153.1, 169.0; IR (KBr, cm $\left.{ }^{-1}\right) 3328$ (s, OH), 2937 (s), 1760 (s, CO), $1597(\mathrm{~m}), 1455(\mathrm{~s}), 1190(\mathrm{~s})$; mass spectrum (EI) $\mathrm{m} / \mathrm{z}$ (\% rel. intensity) $430 \mathrm{M}^{+}$ (55), $388 \mathrm{M}^{+}-\mathrm{CH}_{2} \mathrm{CO}$ (90), $356 \mathrm{M}^{+}-\mathrm{CH}_{2} \mathrm{CO}-\mathrm{MeOH}$ (100), 341 (11), 324 (26), 309 (15), 281 (9); HRMS (EI) calcd for $\mathrm{C}_{24} \mathrm{H}_{30} \mathrm{O}_{7}$ 430.1992, found 430.1988. 


\section{8-Acyloxy-1,2,3,7,11-pentamethoxy-9-propyl-5,6-dihydro-5H-} dibenzo[a,c]cycloheptenes. $\mathbf{R}^{2}=\mathrm{COCH}_{3}(7), \mathrm{COCMe}_{3}(8)$.

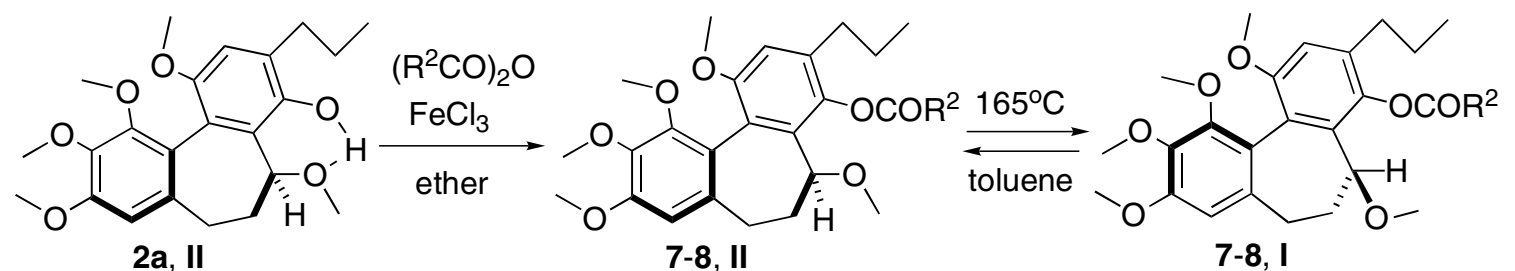

To a stirred suspension of $\left(\mathrm{a} S^{*}, 7 S^{*}\right)-2\left(\mathrm{R}, \mathrm{R}^{1}=\mathrm{Me}\right)^{1}(0.1 \mathrm{~g}, 0.249 \mathrm{mmol})$ in $2 \mathrm{~mL}$ of anhydrous ether under nitrogen was added $(\mathrm{RCO})_{2} \mathrm{O}(1.81 \mathrm{mmol})$ in one portion. Then 10-12 mg of anhydrous $\mathrm{FeCl}_{3}$ was added quickly as a solid. The reaction was monitored by TLC for the disappearance of the starting material ( 1 hour for $\mathrm{R}=\mathrm{COCH}_{3}, 3.5$ hours for $\mathrm{R}=\mathrm{COCMe}_{3}$ ). The reaction was quenched with $2 \mathrm{~mL}$ of the saturated $\mathrm{Na}_{2} \mathrm{HPO}_{4}$ solution, stirred 1 hour, then extracted twice with ether and then the organic layers were combined and dried over $\mathrm{MgSO}_{4}$.

$$
\mathrm{R}^{2}=\mathrm{COCH}_{3}
$$

Solvent was removed in vacuo to give $\left(\mathrm{a} S^{*}, 7 S^{*}\right)-\mathbf{7}, \mathbf{I I}(0.11 \mathrm{~g}, 99 \%)$.

Acetate 7,II: Colorless crystals. M.p. $101.5-102.5^{\circ} \mathrm{C}$.

${ }^{1} \mathrm{H}-\mathrm{NMR}\left(500 \mathrm{MHz}, \mathrm{CDCl}_{3}\right) \delta 1.01(\mathrm{t}, 3 \mathrm{H}, \mathrm{J}=7.4 \mathrm{~Hz}), 1.60-1.74(\mathrm{~m}, 2 \mathrm{H}), 2.05-2.19(\mathrm{~m}$, $2 \mathrm{H}), 2.26(\mathrm{~s}, 3 \mathrm{H}), 2.35-2.40(\mathrm{~m}, 2 \mathrm{H}), 2.54(\mathrm{t}, 2 \mathrm{H}, \mathrm{J}=7.6 \mathrm{~Hz}), 3.29(\mathrm{~s}, 3 \mathrm{H}), 3.74(\mathrm{~s}, 3 \mathrm{H})$, $3.76(\mathrm{~s}, 3 \mathrm{H}), 3.89(\mathrm{~s}, 6 \mathrm{H}), 4.02(\mathrm{dd}, 1 \mathrm{H}, \mathrm{J}=10.9,7.0 \mathrm{~Hz}), 6.56(\mathrm{~s}, 1 \mathrm{H}), 6.80(\mathrm{~s}, 1 \mathrm{H}) ;{ }^{13} \mathrm{C}-$ NMR (125 MHz, $\left.\mathrm{CDCl}_{3}\right) \delta 14.2,20.7,23.2,30.1,33.0,36.8,55.8,55.9,57.8,60.7,60.8$, 81.4, 106.6, 111.1, 119.8, 122.1, 130.0, 135.3, 135.7, 138.8, 140.2, 151.4, 153.0, 153.6, 170.6; IR (mix. of diastereomers) (KBr, cm-1) 2938 (s), 1761 (s, CO), 1465 (s), 1207 (s), 1120 (m); mass spectrum (EI) $\mathrm{m} / \mathrm{z}$ (\% rel. intensity) $444 \mathrm{M}^{+}$(75), 402 (6), 370 (100), 355 (13), 339 (17), 324 (9), 295 (7); HRMS (EI) calcd for $\mathrm{C}_{25} \mathrm{H}_{32} \mathrm{O}_{7}$ 444.2148, found 
444.2151. Anal (mix of diastereomers) calcd for $\mathrm{C}_{25} \mathrm{H}_{32} \mathrm{O}_{7}: \mathrm{C}, 67.55 ; \mathrm{H}, 7.26$. Found $\mathrm{C}$, $67.52 ; \mathrm{H}, 7.39$.

The opposite $\left(\mathrm{a} R^{*}, 7 S^{*}\right)$ diastereomer $\mathbf{7 , I}$ can be obtained from $\mathbf{7 , I I}$ by thermal epimerization $\left(165^{\circ} \mathrm{C}, 30\right.$ hours $)$ according to the described below procedure.

Acetate 7,I: Colorless crystals. M.p. $157.5-158.0^{\circ} \mathrm{C}$.

${ }^{1} \mathrm{H}-\mathrm{NMR}\left(500 \mathrm{MHz}, \mathrm{CDCl}_{3}\right) \delta 1.02(\mathrm{t}, 3 \mathrm{H}, \mathrm{J}=7.4 \mathrm{~Hz}), 1.64-1.73(\mathrm{~m}, 2 \mathrm{H}), 2.13-2.61(\mathrm{~m}$, 4H), $2.34(\mathrm{~s}, 3 \mathrm{H}), 2.49(\mathrm{t}, 2 \mathrm{H}, \mathrm{J}=7.8 \mathrm{~Hz}), 2.87(\mathrm{~s}, 3 \mathrm{H}), 3.79(\mathrm{~s}, 3 \mathrm{H}), 3.82(\mathrm{~s}, 3 \mathrm{H}), 3.85(\mathrm{~s}$, $3 \mathrm{H}), 3.86(\mathrm{~s}, 3 \mathrm{H}), 4.51(\mathrm{~d}, 1 \mathrm{H}, \mathrm{J}=5.9 \mathrm{~Hz}), 6.54(\mathrm{~s}, 1 \mathrm{H}), 6.84(\mathrm{~s}, 1 \mathrm{H}) ;{ }^{13} \mathrm{C}-\mathrm{NMR}(125$ $\left.\mathrm{MHz}, \mathrm{CDCl}_{3}\right) \delta 14.3,20.6,22.9,30.2,33.0,39.5,55.5,55.9,56.0,60.5,60.8,75.0$, $106.1,111.7,121.4,123.6,131.1,133.7,135.8,140.0,140.8,151.6,152.4,154.5,168.9$; IR (mix. of diastereomers) (KBr, cm $\left.{ }^{-1}\right) 2938(\mathrm{~s}), 1761$ (s, CO), 1465 (s), 1207 (s), 1120 (m); mass spectrum (EI) $m / z$ (\% rel. intensity) $444 \mathrm{M}^{+}$(82), 402 (6), 370 (100), 355 (14), 339 (19), 324 (11), 295 (9); HRMS (EI) calcd for $\mathrm{C}_{25} \mathrm{H}_{32} \mathrm{O}_{7} 444.2148$, found 444.2152. Anal (mix of diastereomers) calcd for $\mathrm{C}_{25} \mathrm{H}_{32} \mathrm{O}_{7}$ : C, 67.55; H, 7.26. Found C, 67.52; $\mathrm{H}$, 7.39.

$$
\mathrm{R}^{2}=\mathrm{COCMe}_{3}
$$

The solvent was removed in vacuo and the residue was dried by warming under high vacuum until a constant mass was reached, giving (a $\left.S^{*}, 7 S^{*}\right)-\mathbf{8}$,II $(0.12 \mathrm{~g}, 99 \%)$. Pivalate 8,II: Yellowish oil.

${ }^{1} \mathrm{H}-\mathrm{NMR}\left(600 \mathrm{MHz}, \mathrm{CDCl}_{3}\right) \delta 1.00(\mathrm{t}, 3 \mathrm{H}, \mathrm{J}=7.3 \mathrm{~Hz}), 1.37(\mathrm{~s}, 9 \mathrm{H}), 1.58-1.71(\mathrm{~m}, 2 \mathrm{H})$, 2.03-2.10 (m, 1H), 2.10-2.18 (m, 1H), 2.35-2.40 (m, 2H), 2.47-2.52 (m, 2H), $3.25(\mathrm{~s}$, 3H), 3.73 (s, 3H), 3.75 (s, 3H), 3.89 (s, 3H), $3.90(\mathrm{~s}, 3 \mathrm{H}), 4.02(\mathrm{dd}, 1 \mathrm{H}, \mathrm{J}=11.1,6.9 \mathrm{~Hz})$, $6.56(\mathrm{~s}, 1 \mathrm{H}), 6.78(\mathrm{~s}, 1 \mathrm{H}) ;{ }^{13} \mathrm{C}-\mathrm{NMR}\left(150 \mathrm{MHz}, \mathrm{CDCl}_{3}\right) \delta 14.1,23.6,27.4,30.4,33.3$, 
$36.7,39.1,55.9,56.0,57.9,60.8,60.9,81.9,106.7,111.4,120.0,122.3,130.8,135.3$, $135.9,139.3,140.2,151.5,153.0,153.5,177.8 ;$ IR (mix. of diastereomers) $\left(\mathrm{KBr}, \mathrm{cm}^{-1}\right)$ 2932 (s), 1749 (s, CO), 1466 (s), 1233 (m), 1115 (m); mass spectrum (EI) m/z (\% rel. intensity) $486 \mathrm{M}^{+}$(15), $416(5), 386 \mathrm{M}^{+}-\mathrm{COCMe}_{3}-\mathrm{Me}(4), 370 \mathrm{M}^{+}-\mathrm{COCMe}_{3}-\mathrm{MeO}$ (23), 339 (4), 292 (11), 149 (8), 57 (100). Anal (mix of diastereomers) calcd for $\mathrm{C}_{28} \mathrm{H}_{38} \mathrm{O}_{7}: \mathrm{C}, 69.11 ; \mathrm{H}$, 7.87. Found C, 69.06; H, 7.87.

The opposite $\left(\mathrm{a} R^{*}, 7 S^{*}\right)$ diastereomer $\mathbf{8 , I}$ can be obtained from $\mathbf{8 , I I}$ by thermal epimerization $\left(165^{\circ} \mathrm{C}, 30\right.$ hours) according to the described below procedure.

Pivalate 8,I: Colorless crystals. M.p. $150.9-151.3{ }^{\circ} \mathrm{C}$.

${ }^{1} \mathrm{H}-\mathrm{NMR}\left(300 \mathrm{MHz}, 100^{\circ} \mathrm{C}\right.$, DMSO-d 6 ) $\delta 0.97(\mathrm{t}, 3 \mathrm{H}, \mathrm{J}=7.4 \mathrm{~Hz}), 1.38(\mathrm{~s}, 9 \mathrm{H}), 1.56-1.70$ (m, 2H), 2.05-2.14 (m, 2H), 2.19-2.38 (m, 2H), 2.41-2.48 (m, 2H), $2.77(\mathrm{~s}, 3 \mathrm{H}), 3.65(\mathrm{~s}$, 3H), $3.71(\mathrm{~s}, 3 \mathrm{H}), 3.75(\mathrm{~s}, 3 \mathrm{H}), 3.81(\mathrm{~s}, 3 \mathrm{H}), 4.37-4.40(\mathrm{~m}, 1 \mathrm{H}), 6.57(\mathrm{~s}, 1 \mathrm{H}), 6.93(\mathrm{~s}, 1 \mathrm{H})$; ${ }^{13} \mathrm{C}-\mathrm{NMR}\left(75 \mathrm{MHz}, \mathrm{CDCl}_{3}\right) \delta 14.0,23.3,27.2,30.3,33.0,39.1,55.9,60.6,74.9,106.2$, 112.2, 121.6, 123.8, 131.3, 133.6, 135.7, 140.1, 140.8, 151.7, 152.3, 154.4, 177.8, one $\left(\mathrm{CH}_{2}\right)$ couldn't be located. Based on integration, signals at 55.9 and $60.6 \mathrm{ppm}$ may contain 3 and $2\left(\mathrm{CH}_{3}\right)$, respectively; IR (mix. of diastereomers) $\left(\mathrm{KBr}, \mathrm{cm}^{-1}\right) 2932(\mathrm{~s})$, 1749 (s, CO), 1466 (s), $1233(\mathrm{~m}), 1115(\mathrm{~m})$; mass spectrum (EI) $\mathrm{m} / \mathrm{z}$ (\% rel. intensity) $486 \mathrm{M}^{+}(13), 386 \mathrm{M}^{+}-\mathrm{COCMe}_{3}-\mathrm{Me}(100), 370 \mathrm{M}^{+}-\mathrm{COCMe}_{3}-\mathrm{MeO}$ (16), 355 (15), 339 (15), 323 (9), 192 (5). Anal (mix of diastereomers) calcd for $\mathrm{C}_{28} \mathrm{H}_{38} \mathrm{O}_{7}: \mathrm{C}, 69.11 ; \mathrm{H}$, 7.87. Found C, 69.06; H, 7.87. 


\section{1,2,3,7,8,11-Hexamethoxy-9-propyl-5,6-dihydro-5H-dibenzo[a,c]cycloheptenes (9).}

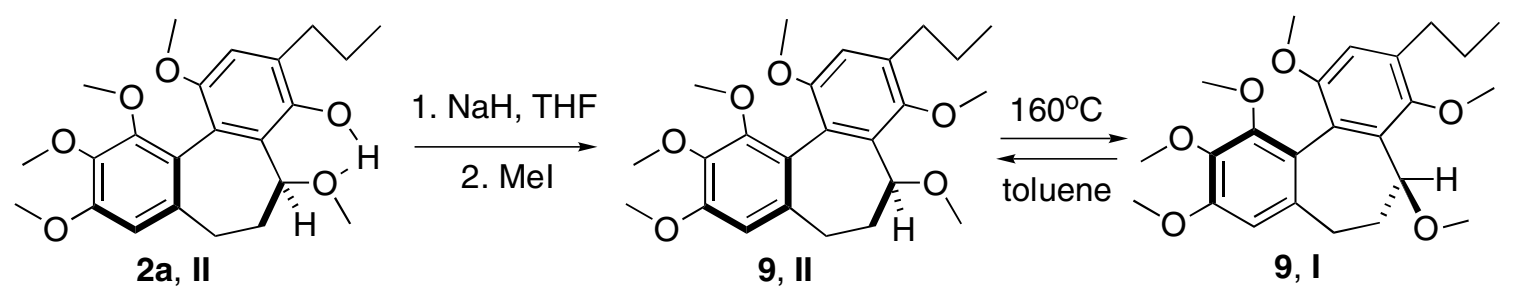

To a stirred solution of $\left(\mathrm{a} S^{*}, 7 S^{*}\right)-\mathbf{2}, \mathbf{I I}\left(\mathrm{R}, \mathrm{R}^{1}=\mathrm{Me}\right)(0.05 \mathrm{~g}, 0.124 \mathrm{mmol})$ in $2 \mathrm{~mL}$ of dry THF under nitrogen was added $\mathrm{NaH}(0.015 \mathrm{~g}, 0.622 \mathrm{mmol})$ and the resulting suspension was refluxed for 3 hours. Then MeI $(0.088 \mathrm{~g}, 0.622 \mathrm{mmol})$ was added in one portion and the mixture was stirred at $65{ }^{\circ} \mathrm{C}$ for $4 \mathrm{~h}$. The reaction mixture was cooled to room temperature and carefully quenched with $5 \mathrm{~mL}$ of water and acidified with $10 \%$ $\mathrm{HCl}$ solution. The product was extracted twice with ether and then the combined organic layers were separated and dried over $\mathrm{CaCl}_{2}$. The solvent was removed and the residue was purified chromatographically (silica gel, $15 \%$ EtOAc in hexane) to give (a $\left.S^{*}, 7 S^{*}\right)$ 9,II (0.045 g, $87 \%)$. Colorless oil.

${ }^{1} \mathrm{H}-\mathrm{NMR}\left(600 \mathrm{MHz}, \mathrm{CDCl}_{3}\right) \delta 1.04(\mathrm{t}, 3 \mathrm{H}, \mathrm{J}=7.4 \mathrm{~Hz}), 1.64-1.77(\mathrm{~m}, 2 \mathrm{H}), 2.08-2.17(\mathrm{~m}$, 1H), 2.30-2.39 (m, 2H), 2.40-2.47 (m, 1H), 2.60 (ddd, 1H, J = 13.7, 9.6, $6.0 \mathrm{~Hz},), 2.75$ (ddd, 1H, J = 13.7, 9.9, $6.1 \mathrm{~Hz}), 3.32(\mathrm{~s}, 3 \mathrm{H}), 3.70(\mathrm{~s}, 3 \mathrm{H}), 3.74(\mathrm{~s}, 3 \mathrm{H}), 3.77$ (s, 3H), $3.892(\mathrm{~s}, 3 \mathrm{H}), 3.895(\mathrm{~s}, 3 \mathrm{H}), 4.06(\mathrm{dd}, 1 \mathrm{H}, \mathrm{J}=11.8,5.9 \mathrm{~Hz}), 6.56(\mathrm{~s}, 1 \mathrm{H}), 6.77(\mathrm{~s}, 1 \mathrm{H})$; ${ }^{13} \mathrm{C}-\mathrm{NMR}\left(75 \mathrm{MHz}, \mathrm{CDCl}_{3}\right) \delta 14.3,24.0,30.4,32.5,38.9,55.9,56.1,58.2,60.7,60.9$, $62.5,81.3,106.6,111.4,120.8,122.1,130.6,135.2,136.9,140.4,149.1,151.5,152.1$, 152.9; IR (mix. of diastereomers) $\left(\mathrm{KBr}, \mathrm{cm}^{-1}\right) 2937$ (s), 1600 (m), 1465 (s), 1238 (s), 1093 (s); mass spectrum (EI) $m / z$ (\% rel. intensity) $416 \mathrm{M}^{+}$(100), $385 \mathrm{M}^{+}-\mathrm{MeO}(11)$, $369 \mathrm{M}^{+}-\mathrm{MeOH}-\mathrm{Me}(11), 353$ (7), 339 (7), 167 (9), 149 (29), 57 (11). Anal (mix of diastereomers) calcd for $\mathrm{C}_{24} \mathrm{H}_{32} \mathrm{O}_{6}$ : C, 69.21; H, 7.74. Found C, 69.03; H, 7.81. 


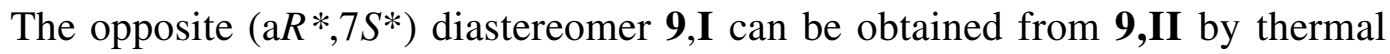
epimerization $\left(160{ }^{\circ} \mathrm{C}, 20\right.$ hours) according to the described below procedure. Colorless crystals. M.p. $126.6-127.0^{\circ} \mathrm{C}$.

${ }^{1} \mathrm{H}-\mathrm{NMR}\left(300 \mathrm{MHz}, \mathrm{CDCl}_{3}\right) \delta 1.05(\mathrm{t}, 3 \mathrm{H}, \mathrm{J}=7.4 \mathrm{~Hz}), 1.67-1.81(\mathrm{~m}, 2 \mathrm{H}), 2.22-2.55(\mathrm{~m}$, 4H), 2.60-2.76 (m, 2H), $2.92(\mathrm{~s}, 3 \mathrm{H}), 3.72(\mathrm{~s}, 3 \mathrm{H}), 3.77$ (s, 3H), $3.81(\mathrm{~s}, 3 \mathrm{H}), 3.85(\mathrm{~s}, 3 \mathrm{H})$, $3.87(\mathrm{~s}, 3 \mathrm{H}), 4.95(\mathrm{dd}, 1 \mathrm{H}, \mathrm{J}=5.3,1.8 \mathrm{~Hz}), 6.55(\mathrm{~s}, 1 \mathrm{H}), 6.80(\mathrm{~s}, 1 \mathrm{H}) ;{ }^{13} \mathrm{C}-\mathrm{NMR}(75$ $\left.\mathrm{MHz}, \mathrm{CDCl}_{3}\right) \delta 14.3,23.8,30.4,32.1,40.3,55.5,55.85,55.93,60.5,60.7,62.0,74.4$, $106.1,111.8,121.9,123.5,131.7,134.6,135.6,140.0,150.0,151.6,152.1,153.0 ;$ IR (mix. of diastereomers) $\left(\mathrm{KBr}, \mathrm{cm}^{-1}\right) 2937$ (s), 1600 (m), 1465 (s), 1238 (s), 1093 (s); mass spectrum (EI) $\mathrm{m} / \mathrm{z}$ (\% rel. intensity) $416 \mathrm{M}^{+}$(100), $385 \mathrm{M}^{+}-\mathrm{MeO}(8), 369 \mathrm{M}^{+}-$ MeOH - Me (8), 254 (27), 239 (8), 200 (8), 149 (16), 57 (34). Anal (mix of diastereomers) calcd for $\mathrm{C}_{24} \mathrm{H}_{32} \mathrm{O}_{6}$ : C, 69.21; H, 7.74. Found C, 69.03; H, 7.81.

\section{1,2,3,7,11-Pentamethoxy-8-trialkylsiloxy-9-propyl-5,6-dihydro-5 $H$ -}

dibenzo[a,c]cycloheptenes. PG = TMS (10), TBDMS (11), TIPS (12); R = Me, Et, $i$ -

Pr. $\mathbf{R}^{1}=$ Me, Et, $i$-Pr, $t$-Bu. General Procedure.

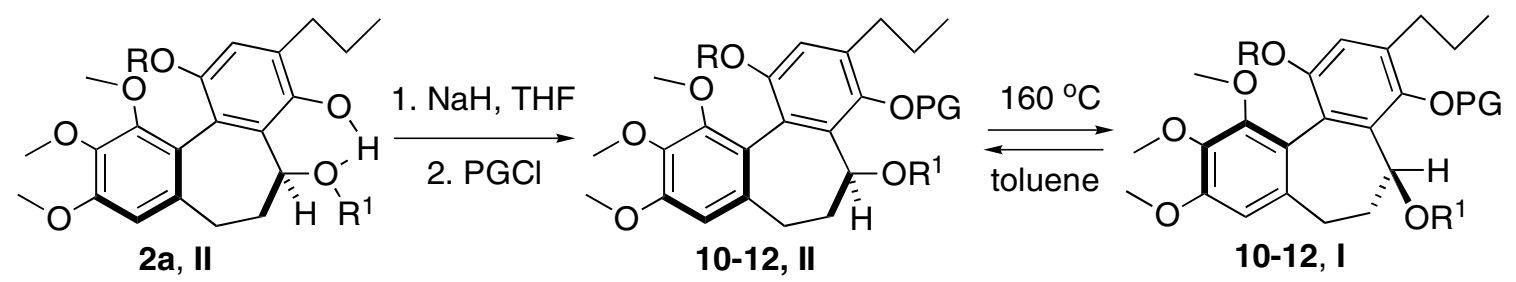

To a stirred solution of the proper $\left(\mathrm{a} S^{*}, 7 S^{*}\right)$ phenol 2,II (1 eq.) in dry THF ( $0.065 \mathrm{M})$ under nitrogen was added $\mathrm{NaH}(3 \mathrm{eq})$ as a $60 \%$ suspension in mineral oil and the mixture was refluxed for 3 hours. Then it was treated with the desired silyl chloride PGCl (3 eq) under the conditions given below. The progress of the reaction was 
monitored by TLC. After the protection was complete, the mixture was carefully quenched with saturated aqueous $\mathrm{NaHCO}_{3}$ at room temperature, the product was extracted twice with ether, and then the organic layers were separated and dried over $\mathrm{CaCl}_{2}$. The solvent was removed and the residue was purified on a short column (silica gel, $15 \%$ EtOAc in hexane). The products and yields are given below:

$$
\mathrm{R}, \mathrm{R}^{1}=\mathrm{Me}:
$$

Protected phenol 10: $\mathrm{PG}=$ TMS: Protection conditions: TMSCl was added at r.t. and the mixture was stirred for 0.5 hours. Purification provided the TMS protected $\left(\mathrm{a} S^{*}, 7 S^{*}\right)$ phenol 10,II (90\%). Yellowish oil.

${ }^{1} \mathrm{H}-\mathrm{NMR}\left(600 \mathrm{MHz}, \mathrm{CDCl}_{3}\right) \delta 0.25(\mathrm{~s}, 9 \mathrm{H}), 1.01(\mathrm{t}, 3 \mathrm{H}, \mathrm{J}=7.4 \mathrm{~Hz}), 1.60-1.68(\mathrm{~m}, 1 \mathrm{H})$, 1.68-1.76 (m, 1H), 2.02-2.08 (m, 1H), 2.21-2.33 (m, 2H), 2.38-2.47 (m, 2H), 2.76 (ddd, $1 \mathrm{H}, \mathrm{J}=14.0,10.2,5.7 \mathrm{~Hz}), 3.22(\mathrm{~s}, 3 \mathrm{H}), 3.67(\mathrm{~s}, 3 \mathrm{H}), 3.71(\mathrm{~s}, 3 \mathrm{H}), 3.89(\mathrm{~s}, 3 \mathrm{H}), 3.90(\mathrm{~s}$, 3H), $4.02(\mathrm{dd}, 1 \mathrm{H}, \mathrm{J}=11.4,6.2 \mathrm{~Hz}), 6.55(\mathrm{~s}, 1 \mathrm{H}), 6.73(\mathrm{~s}, 1 \mathrm{H}) ;{ }^{13} \mathrm{C}-\mathrm{NMR}(150 \mathrm{MHz}$, $\left.\mathrm{CDCl}_{3}\right) \delta 1.3,14.1,22.9,30.5,33.9,38.1,55.9,56.1,57.3,60.6,60.9,81.2,106.6,111.4$, $121.0,122.0,128.4,134.4,135.2,140.3,143.9,150.4,151.5,152.7 ;$ IR (mix. of diastereomers) $\left(\mathrm{KBr}, \mathrm{cm}^{-1}\right) 2937(\mathrm{~s}), 1600(\mathrm{~m}), 1465(\mathrm{~s}), 1236(\mathrm{~s}), 1107$ (s); mass spectrum (EI) $m / z$ (\% rel. intensity) $474 \mathrm{M}^{+}$(100), $443 \mathrm{M}^{+}-\mathrm{MeO}(13), 370 \mathrm{M}^{+}-\mathrm{MeO}-$ $\mathrm{SiMe}_{3}$ (89), 355 (10), 339 (15), 262 (16), 233 (16), 149 (73), 57 (25). Anal (mix of diastereomers) calcd for $\mathrm{C}_{26} \mathrm{H}_{38} \mathrm{O}_{6} \mathrm{Si}: \mathrm{C}, 65.79 ; \mathrm{H}$, 8.07. Found C, 65.69; H, 8.10.

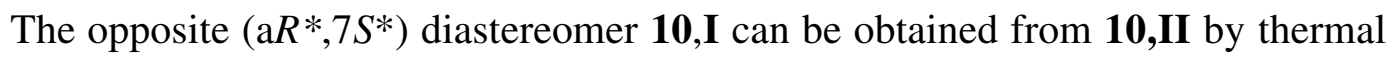
epimerization $\left(160{ }^{\circ} \mathrm{C}, 20\right.$ hours) according to the described below procedure. Colorless crystals. M.p. $138.0-139.2^{\circ} \mathrm{C}$. 
${ }^{1} \mathrm{H}-\mathrm{NMR}\left(600 \mathrm{MHz}, \mathrm{CDCl}_{3}\right) \delta 0.27(\mathrm{~s}, 9 \mathrm{H}), 1.02(\mathrm{t}, 3 \mathrm{H}, \mathrm{J}=7.4 \mathrm{~Hz}), 1.63-1.75(\mathrm{~m}, 2 \mathrm{H})$, 2.20-2.29 (m, 2H), 2.33-2.39 (m, 1H), $2.46(\mathrm{td}, 1 \mathrm{H}, \mathrm{J}=12.7,7.5 \mathrm{~Hz}), 2.52(\mathrm{ddd}, 1 \mathrm{H}, \mathrm{J}=$ 14.1, 9.6, 6.3 Hz), 2.64 (ddd, 1H, J = 14.1, 9.7, $6.2 \mathrm{~Hz}$ ), 2.88 (s, 3H), 3.73 (s, 3H), 3.76 (s, 3H), $3.855(\mathrm{~s}, 3 \mathrm{H}), 3.862(\mathrm{~s}, 3 \mathrm{H}), 4.89(\mathrm{dd}, 1 \mathrm{H}, \mathrm{J}=5.3,1.7 \mathrm{~Hz}), 6.52(\mathrm{~s}, 1 \mathrm{H}), 6.75(\mathrm{~s}$, $1 \mathrm{H}) ;{ }^{13} \mathrm{C}-\mathrm{NMR}\left(150 \mathrm{MHz}, \mathrm{CDCl}_{3}\right) \delta 0.9,14.1,22.9,30.5,33.9,39.6,55.6,55.7,56.2$, $60.6,60.7,74.5,106.1,112.1,122.4,123.4,129.6,132.1,135.5,140.2,145.5,151.4$, 151.8, 152.1; IR (mix. of diastereomers) $\left(\mathrm{KBr}, \mathrm{cm}^{-1}\right) 2937$ (s), 1600 (m), 1465 (s), 1236 (s), 1107 (s); mass spectrum (EI) $\mathrm{m} / z$ (\% rel. intensity) $474 \mathrm{M}^{+}(30), 442 \mathrm{M}^{+}-\mathrm{MeOH}$ (27), $370 \mathrm{M}^{+}-\mathrm{MeO}-\mathrm{SiMe}_{3}$ (38), 239 (51), 226 (22), 172 (25), 159 (100), 133 (61), 79 (44). Anal (mix of diastereomers) calcd for $\mathrm{C}_{26} \mathrm{H}_{38} \mathrm{O}_{6} \mathrm{Si}: \mathrm{C}, 65.79 ; \mathrm{H}, 8.07$. Found $\mathrm{C}$, $65.69 ; \mathrm{H}, 8.10$.

Protected phenol 11: $\mathrm{PG}=$ TBDMS: Protection conditions: $\mathrm{TBDMSCl}$ was added at r.t. and the mixture was stirred for 2.5 hours. Purification provided the TBDMS protected (a $\left.S^{*}, 7 S^{*}\right)$ phenol 11,II $(92 \%)$. Yellowish oil.

${ }^{1} \mathrm{H}-\mathrm{NMR}\left(600 \mathrm{MHz}, \mathrm{CDCl}_{3}\right) \delta 0.17(\mathrm{~s}, 3 \mathrm{H}), 0.26(\mathrm{~s}, 3 \mathrm{H}), 0.93(\mathrm{t}, 3 \mathrm{H}, \mathrm{J}=7.3 \mathrm{~Hz}), 1.04(\mathrm{~s}$, $9 \mathrm{H}), 1.54-1.66(\mathrm{~m}, 2 \mathrm{H}), 2.16-2.28(\mathrm{~m}, 2 \mathrm{H}), 2.35-2.39(\mathrm{~m}, 1 \mathrm{H}), 2.40-2.44(\mathrm{~m}, 2 \mathrm{H}), 2.92$ (ddd, 1H, J = 13.7, 9.2, $6.6 \mathrm{~Hz}$ ), 3.23 (s, 3H), 3.57 (s, 3H), $3.71(\mathrm{~s}, 3 \mathrm{H}), 3.893(\mathrm{~s}, 3 \mathrm{H})$, $3.895(\mathrm{~s}, 3 \mathrm{H}), 4.00(\mathrm{dd}, 1 \mathrm{H}, \mathrm{J}=11.3,6.9 \mathrm{~Hz}), 6.56(\mathrm{~s}, 1 \mathrm{H}), 6.72(\mathrm{~s}, 1 \mathrm{H}) ;{ }^{13} \mathrm{C}-\mathrm{NMR}(150$ $\left.\mathrm{MHz}, \mathrm{CDCl}_{3}\right) \delta$-3.1, -1.6, 13.7, 18.8, 23.9, 26.5, 30.6, 33.3, 38.2, 55.9, 56.2, 57.8, 60.5, $61.0,82.1,106.5,111.5,121.2,122.0,128.0,134.5,135.4,140.3,143.4,150.6,151.5$, 152.7; IR (mix. of diastereomers) $\left(\mathrm{KBr}, \mathrm{cm}^{-1}\right) 2935$ (s), 1599 (m), 1464 (s), 1236 (s), 1105 (s); mass spectrum (EI) $m / z$ (\% rel. intensity) $516 \mathrm{M}^{+}(49), 459 \mathrm{M}^{+}-t$-Bu (72), 431 (59), $427 \mathrm{M}^{+}-\mathrm{MeOH}-t$-Bu (100), $412 \mathrm{M}^{+}-\mathrm{MeOH}-t-\mathrm{Bu}-\mathrm{Me}(46), 370 \mathrm{M}^{+}-\mathrm{MeO}-$ 
$t$ - $\mathrm{BuMe}_{2} \mathrm{Si}$ (29), 292 (18), 221 (17), 95 (26). Anal (mix of diastereomers) calcd for $\mathrm{C}_{29} \mathrm{H}_{44} \mathrm{O}_{6} \mathrm{Si}$ : C, 67.41; H, 8.58. Found C, 67.42; H, 8.68.

The opposite $\left(\mathrm{a} R^{*}, 7 S^{*}\right)$ diastereomer $\mathbf{1 1}, \mathbf{I}$ can be obtained from it by thermal epimerization $\left(160{ }^{\circ} \mathrm{C}, 20\right.$ hours) according to the described below procedure. Colorless crystals. M.p. $124.9-126.0^{\circ} \mathrm{C}$.

${ }^{1} \mathrm{H}-\mathrm{NMR}\left(600 \mathrm{MHz}, \mathrm{CDCl}_{3}\right) \delta 0.20(\mathrm{~s}, 3 \mathrm{H}), 0.23(\mathrm{~s}, 3 \mathrm{H}), 0.96(\mathrm{t}, 3 \mathrm{H}, \mathrm{J}=7.4 \mathrm{~Hz}), 1.05(\mathrm{~s}$, $9 \mathrm{H}), 1.62-1.69(\mathrm{~m}, 2 \mathrm{H}), 2.20(\mathrm{td}, 1 \mathrm{H}, \mathrm{J}=14.2,5.2 \mathrm{~Hz}), 2.32-2.39(\mathrm{~m}, 2 \mathrm{H}), 2.47-2.58(\mathrm{~m}$, 2H), 2.71-2.77 (m, 1H), 2.87 (s, 3H), $3.66(\mathrm{~s}, 3 \mathrm{H}), 3.72(\mathrm{~s}, 3 \mathrm{H}), 3.86(\mathrm{~s}, 6 \mathrm{H}), 4.92(\mathrm{~d}, 1 \mathrm{H}$, $\mathrm{J}=6.5 \mathrm{~Hz}), 6.53(\mathrm{~s}, 1 \mathrm{H}), 6.76(\mathrm{~s}, 1 \mathrm{H}) ;{ }^{13} \mathrm{C}-\mathrm{NMR}\left(150 \mathrm{MHz}, \mathrm{CDCl}_{3}\right) \delta-3.0,-2.8,13.8$, $18.8,23.6,26.3,30.7,33.2,39.1,55.5,55.6,56.3,60.6,60.7,74.1,106.0,112.3,122.5$, 123.6, 129.5, 132.0, 135.5, 140.2, 145.1, 151.5, 151.9, 152.1; IR (mix. of diastereomers) $\left(\mathrm{KBr}, \mathrm{cm}^{-1}\right) 2935(\mathrm{~s}), 1599(\mathrm{~m}), 1464(\mathrm{~s}), 1236(\mathrm{~s}), 1105(\mathrm{~s})$; mass spectrum (EI) $\mathrm{m} / \mathrm{z}(\%$ rel. intensity) $516 \mathrm{M}^{+}(53), 459 \mathrm{M}^{+}-t$-Bu (40), 431 (53), $427 \mathrm{M}^{+}$- $\mathrm{MeOH}-t$-Bu (100), $412 \mathrm{M}^{+}-\mathrm{MeOH}-t-\mathrm{Bu}-\mathrm{Me}(49), 370 \mathrm{M}^{+}-\mathrm{MeO}-t-\mathrm{BuMe}_{2} \mathrm{Si}$ (28), 339 (10), 221 (26), 149 (16), 73 (46). Anal (mix of diastereomers) calcd for $\mathrm{C}_{29} \mathrm{H}_{44} \mathrm{O}_{6} \mathrm{Si}$ : C, 67.41; H, 8.58. Found C, 67.42; H, 8.68.

$$
P G=\text { TIPS: }
$$

Protection conditions: TIPSCl was added and the mixture was refluxed for 3 hours $\left(9\right.$ hours if $\mathrm{R}^{1}=t$-Bu). Purification provided the TIPS protected $\left(\mathrm{a} S^{*}, 7 S^{*}\right)$ phenols 12,II. Opposite $\left(\mathrm{a} R^{*}, 7 S^{*}\right)$ diastereomers 12 ,I can be obtained from them by thermal epimerization $\left(160^{\circ} \mathrm{C}, 20\right.$ hours $)$ according to the described below procedure. 
Protected phenol 12a: $\mathrm{R}, \mathrm{R}^{1}=\mathrm{Me}\left(\mathrm{a} S^{*}, 7 S^{*}\right)$ diastereomer 12a,II: Yield $97 \%$. Colorless crystals. M.p. $139.4-140.5^{\circ} \mathrm{C}$.

${ }^{1} \mathrm{H}-\mathrm{NMR}\left(600 \mathrm{MHz}, \mathrm{CDCl}_{3}\right) \delta 0.97(\mathrm{t}, 3 \mathrm{H}, \mathrm{J}=7.3 \mathrm{~Hz}), 1.12(\mathrm{~d}, 9 \mathrm{H}, \mathrm{J}=7.5 \mathrm{~Hz}), 1.13(\mathrm{~d}$, 9H, J = 7.5 Hz), 1.39 (sept., 3H, J = 7.5 Hz), 1.57-1.68 (m, 2H), 2.19-2.25 (m, 2H), 2.34$2.43(\mathrm{~m}, 3 \mathrm{H}), 2.88(\mathrm{ddd}, 1 \mathrm{H}, \mathrm{J}=13.7,9.5,6.1 \mathrm{~Hz}), 3.23(\mathrm{~s}, 3 \mathrm{H}), 3.58(\mathrm{~s}), 3.70(\mathrm{~s}, 3 \mathrm{H})$, $3.89(\mathrm{~s}, 3 \mathrm{H}), 3.90(\mathrm{~s}, 3 \mathrm{H}), 4.01(\mathrm{dd}, 1 \mathrm{H}, \mathrm{J}=9.9,8.7 \mathrm{~Hz}), 6.55(\mathrm{~s}, 1 \mathrm{H}), 6.70(\mathrm{~s}, 1 \mathrm{H}) ;{ }^{13} \mathrm{C}-$ NMR $\left(75 \mathrm{MHz}, \mathrm{CDCl}_{3}\right) \delta 13.9,14.6,18.3,18.4,24.3,30.7,33.6,37.9,55.9,56.3,57.7$, $60.5,61.0,82.1,106.8,111.5,121.3,122.2,127.6,133.9,135.4,140.4,144.9,150.4$ 151.6, 152.7; IR (mix. of diastereomers) $\left(\mathrm{KBr}, \mathrm{cm}^{-1}\right) 2935$ (s), 1599 (m), 1462 (s), 1239 (s), 1105 (s); mass spectrum (EI) $m / z$ (\% rel. intensity) $558 \mathrm{M}^{+}$(76), $526 \mathrm{M}^{+}-\mathrm{MeOH}$ (15), $515 \mathrm{M}^{+}-i$-Pr (33), $483 \mathrm{M}^{+}-\mathrm{MeOH}-i$-Pr (100), $468 \mathrm{M}^{+}-\mathrm{MeOH}-i$-Pr $-\mathrm{Me}$ (37), 452 (14), $370 \mathrm{M}^{+}-\mathrm{MeO}-\mathrm{Si}(i-\operatorname{Pr})_{3}(28), 149$ (9), 89 (15), 75 (13). Anal (mix of diastereomers) calcd for $\mathrm{C}_{32} \mathrm{H}_{50} \mathrm{O}_{6} \mathrm{Si}: \mathrm{C}, 68.78 ; \mathrm{H}, 9.02$. Found C, 68.84; H, 9.05. $\left(\mathrm{a} R^{*}, 7 S^{*}\right)$ diastereomer 12a,I: Colorless crystals. M.p. $150.9-152.6{ }^{\circ} \mathrm{C}$.

${ }^{1} \mathrm{H}-\mathrm{NMR}\left(600 \mathrm{MHz}, \mathrm{CDCl}_{3}\right) \delta 1.00(\mathrm{t}, 3 \mathrm{H}, \mathrm{J}=7.5 \mathrm{~Hz}), 1.14(\mathrm{~d}, 9 \mathrm{H}, \mathrm{J}=7.6 \mathrm{~Hz}), 1.15(\mathrm{~d}$, $9 \mathrm{H}, \mathrm{J}=7.5 \mathrm{~Hz}), 1.30$ (sept., $3 \mathrm{H}, \mathrm{J}=7.6 \mathrm{~Hz}), 1.61-1.73(\mathrm{~m}, 2 \mathrm{H}), 2.23(\mathrm{td}, 1 \mathrm{H}, \mathrm{J}=14.1$, $5.3 \mathrm{~Hz}), 2.30-2.37$ (m, 2H), 2.47-2.54 (m, 2H), 2.77 (ddd, 1H, J = 14.0, 9.6, $6.5 \mathrm{~Hz}), 2.89$ (s, 3H), 3.67 (s, 3H), $3.72(\mathrm{~s}, 3 \mathrm{H}), 3.87(\mathrm{~s}, 6 \mathrm{H}), 4.92(\mathrm{~d}, 1 \mathrm{H}, \mathrm{J}=6.3 \mathrm{~Hz}), 6.52(\mathrm{~s}, 1 \mathrm{H})$, $6.74(\mathrm{~s}, 1 \mathrm{H}) ;{ }^{13} \mathrm{C}-\mathrm{NMR}\left(75 \mathrm{MHz}, \mathrm{CDCl}_{3}\right) \delta 14.0,14.4,18.20,18.23,23.8,30.7,33.3$, $39.1,55.5,55.6,56.3,60.6,60.7,74.2,106.1,112.3,122.7,123.6,129.2,131.6,135.3$, 140.3, 146.3, 151.3, 151.9, 152.0; IR (mix. of diastereomers) $\left(\mathrm{KBr}, \mathrm{cm}^{-1}\right) 2935$ (s), 1599 (m), 1462 (s), 1239 (s), 1105 (s); mass spectrum (EI) $\mathrm{m} / z$ (\% rel. intensity) $558 \mathrm{M}^{+}$(87), $515 \mathrm{M}^{+}-i$-Pr (32), $483 \mathrm{M}^{+}-\mathrm{MeOH}-i-\operatorname{Pr}(100), 468 \mathrm{M}^{+}-\mathrm{MeOH}-i-\mathrm{Pr}-\mathrm{Me}$ (40), 452 
(14), $370 \mathrm{M}^{+}-\mathrm{MeO}-\mathrm{Si}(i-\operatorname{Pr})_{3}(22), 159$ (29), 133 (18), 91 (15). Anal (mix of diastereomers) calcd for $\mathrm{C}_{32} \mathrm{H}_{50} \mathrm{O}_{6} \mathrm{Si}$ C, 68.78; H, 9.02. Found C, 68.84; H, 9.05.

Protected phenol 12b: $\mathrm{R}=\mathrm{Me}, \mathrm{R}^{1}=\mathrm{Et}\left(\mathrm{a} S^{*}, 7 S^{*}\right)$ diastereomer 12b,II: Yield 95 $\%$. Colorless crystals. M.p. $128.8-129.4{ }^{\circ} \mathrm{C}$.

${ }^{1} \mathrm{H}-\mathrm{NMR}\left(600 \mathrm{MHz}, \mathrm{CDCl}_{3}\right) \delta 0.95(\mathrm{t}, 3 \mathrm{H}, \mathrm{J}=7.4 \mathrm{~Hz}), 1.09(\mathrm{t}, 3 \mathrm{H}, \mathrm{J}=7.1 \mathrm{~Hz}), 1.12(\mathrm{~d}$, 9H, J = 7.5 Hz), $1.13(\mathrm{~d}, 9 \mathrm{H}, \mathrm{J}=7.5 \mathrm{~Hz}), 1.38($ sept., 3H, J = 7.5 Hz), 1.54-1.66 (m, 2H), 2.16-2.29 (m, 2H), 2.34-2.44 (m, 3H), 2.89 (ddd, $1 \mathrm{H}, \mathrm{J}=13.5,9.5,6.3 \mathrm{~Hz}), 3.06-3.13(\mathrm{~m}$, 1H), $3.57(\mathrm{~s}, 3 \mathrm{H}), 3.66-3.72(\mathrm{~m}, 1 \mathrm{H}), 3.70(\mathrm{~s}, 3 \mathrm{H}), 3.89(\mathrm{~s}, 3 \mathrm{H}), 3.90(\mathrm{~s}, 3 \mathrm{H}), 4.12(\mathrm{dd}$, $1 \mathrm{H}, \mathrm{J}=11.6,6.9 \mathrm{~Hz}), 6.55(\mathrm{~s}, 1 \mathrm{H}), 6.68(\mathrm{~s}, 1 \mathrm{H}) ;{ }^{13} \mathrm{C}-\mathrm{NMR}\left(75 \mathrm{MHz}, \mathrm{CDCl}_{3}\right) \delta 13.8$, $14.6,15.2,18.4,18.5,24.4,30.9,33.8,38.1,55.9,56.3,60.5,61.0,65.3,80.2,106.9$, $111.4,121.4,122.2,128.5,133.7,135.6,140.4,145.3,150.4,151.6,152.7$; IR (mix. of diastereomers) $\left(\mathrm{KBr}, \mathrm{cm}^{-1}\right) 2937(\mathrm{~s}), 1596(\mathrm{~m}), 1465(\mathrm{~s}), 1231(\mathrm{~s}), 1101(\mathrm{~s})$; mass spectrum (EI) $m / z$ (\% rel. intensity) $572 \mathrm{M}^{+}(93), 526 \mathrm{M}^{+}$- EtOH (100), $483 \mathrm{M}^{+}$- EtOH $i-\operatorname{Pr}(94), 468 \mathrm{M}^{+}-\mathrm{EtOH}-i-\operatorname{Pr}-\mathrm{Me}(37), 452$ (37), $370 \mathrm{M}^{+}-\mathrm{EtO}-\mathrm{Si}(i-\operatorname{Pr})_{3}(25), 159$ (13), 59 (19), 43 (47). Anal (mix of diastereomers) calcd for $\mathrm{C}_{33} \mathrm{H}_{52} \mathrm{O}_{6} \mathrm{Si}$ : C, 69.19; H, 9.15. Found C, 69.17; H, 9.16.

$\left(\mathrm{a} R^{*}, 7 S^{*}\right)$ diastereomer 12b,I: Colorless crystals. M.p. $162.1-162.5^{\circ} \mathrm{C}$.

${ }^{1} \mathrm{H}-\mathrm{NMR}\left(300 \mathrm{MHz}, \mathrm{CDCl}_{3}\right) \delta 0.79(\mathrm{t}, 3 \mathrm{H}, \mathrm{J}=7.0 \mathrm{~Hz}), 1.00(\mathrm{t}, 3 \mathrm{H}, \mathrm{J}=7.4 \mathrm{~Hz}), 1.13(\mathrm{~d}$, $18 \mathrm{H}, \mathrm{J}=7.5 \mathrm{~Hz}), 1.22-1.35(\mathrm{~m}, 3 \mathrm{H}), 1.59-1.73(\mathrm{~m}, 2 \mathrm{H}), 2.19-2.28(\mathrm{~m}, 2 \mathrm{H}), 2.29-2.38(\mathrm{~m}$, $1 \mathrm{H}), 2.44-2.59(\mathrm{~m}, 2 \mathrm{H}), 2.65-2.77(\mathrm{~m}, 1 \mathrm{H}), 2.94-3.05(\mathrm{~m}, 1 \mathrm{H}), 3.06-3.17(\mathrm{~m}, 1 \mathrm{H}), 3.72$ (s, 3H), 3.74 (s, 3H), 3.83 (s, 3H), 3.87 (s, 3H), $5.00(\mathrm{dd}, 1 \mathrm{H}, \mathrm{J}=4.2,2.9 \mathrm{~Hz}), 6.53(\mathrm{~s}$, 1H), $6.72(\mathrm{~s}, 1 \mathrm{H}) ;{ }^{13} \mathrm{C}-\mathrm{NMR}\left(75 \mathrm{MHz}, \mathrm{CDCl}_{3}\right) \delta$ 14.0, 14.4, 15.0, 18.2, 23.7, 30.5, 33.4, $38.2,55.7,56.1,60.4,60.5,62.4,72.3,106.2,111.6,122.9,123.5,129.9,131.5,135.4$, 
$140.2,145.6,151.1,151.6,151.9$, based on integration, signal at $18.2 \mathrm{ppm}$ may contain 2 $\left(\mathrm{CH}_{3}\right)$; IR (mix. of diastereomers) $\left(\mathrm{KBr}, \mathrm{cm}^{-1}\right) 2937$ (s), 1596 (m), 1465 (s), 1231 (s), 1101 (s); mass spectrum (EI) $\mathrm{m} / z$ (\% rel. intensity) $572 \mathrm{M}^{+}$(100), $529(11), 483 \mathrm{M}^{+}$$\mathrm{EtOH}-i-\mathrm{Pr}(43), 468 \mathrm{M}^{+}-\mathrm{EtOH}-i-\mathrm{Pr}-\mathrm{Me}(20), 452(9), 370 \mathrm{M}^{+}-\mathrm{EtO}-\mathrm{Si}(i-\operatorname{Pr})_{3}(12)$, 236 (9), 188 (15), 129 (13), 91 (11). Anal (mix of diastereomers) calcd for $\mathrm{C}_{33} \mathrm{H}_{52} \mathrm{O}_{6} \mathrm{Si}$ : C, 69.19; H, 9.15. Found C, 69.17; H, 9.16.

Protected phenol 12c: $\mathrm{R}=\mathrm{Me}, \mathrm{R}^{1}=i$-Pr $\left(a S^{*}, 7 S^{*}\right)$ diastereomer 12c,II: Yield 93 $\%$. Colorless crystals. M.p. $128.6-129.7{ }^{\circ} \mathrm{C}$.

${ }^{1} \mathrm{H}-\mathrm{NMR}\left(300 \mathrm{MHz}, \mathrm{CDCl}_{3}\right.$ ) $\delta$ 0.90-0.97 (m, 6H), 1.08-1.17 (m, 21H), 1.37 (sept., 3H, J $=7.6 \mathrm{~Hz}), 1.51-1.67(\mathrm{~m}, 2 \mathrm{H}), 2.03-2.18(\mathrm{~m}, 1 \mathrm{H}), 2.21-2.46(\mathrm{~m}, 4 \mathrm{H}), 2.89(\mathrm{ddd}, 1 \mathrm{H}, \mathrm{J}=$ 13.5, 8.8, $6.8 \mathrm{~Hz}$ ), 3.44 (sept., 1H, J = 6.3 Hz), 3.57 (s, 3H), 3.69 (s, 3H), 3.896 (s, 3H), $3.902(\mathrm{~s}, 3 \mathrm{H}), 4.15(\mathrm{dd}, 1 \mathrm{H}, \mathrm{J}=11.5,6.6 \mathrm{~Hz}), 6.55(\mathrm{~s}, 1 \mathrm{H}), 6.65(\mathrm{~s}, 1 \mathrm{H}) ;{ }^{13} \mathrm{C}-\mathrm{NMR}(75$ $\left.\mathrm{MHz}, \mathrm{CDCl}_{3}\right) \delta 13.8,14.5,18.4,18.6,21.9,22.8,24.6,31.1,34.0,38.5,55.9,56.4,60.6$, 61.0, 71.8, 78.1, 107.1, 111.4, 121.6, 122.1, 130.0, 133.7, 135.7, 140.4, 145.9, 150.2, 151.7, 152.6; IR (mix. of diastereomers) (KBr, cm $\left.{ }^{-1}\right) 2964$ (s), 1597 (m), 1463 (s), 1236 (s), 1104 (s); mass spectrum (EI) $m / z$ (\% rel. intensity) $586 \mathrm{M}^{+}(100), 526 \mathrm{M}^{+}-i$-PrOH (12), 501 (17), 485 (22), $483 \mathrm{M}^{+}$- $i-\mathrm{PrOH}-i$-Pr (18), 470 (14), 239 (28), 159 (49), 146 (25), 133 (29), 79 (17). Anal (mix of diastereomers) calcd for $\mathrm{C}_{34} \mathrm{H}_{54} \mathrm{O}_{6} \mathrm{Si}$ : C, 69.58; H, 9.27. Found C, 69.64; H, 9.27.

$\left(\mathrm{a} R^{*}, 7 S^{*}\right)$ diastereomer 12c,I: Colorless crystals. M.p. $149.2-150.0^{\circ} \mathrm{C}$.

${ }^{1} \mathrm{H}-\mathrm{NMR}\left(300 \mathrm{MHz}, \mathrm{CDCl}_{3}\right) \delta 0.72(\mathrm{~d}, 3 \mathrm{H}, \mathrm{J}=6.3 \mathrm{~Hz}), 0.87(\mathrm{~d}, 3 \mathrm{H}, \mathrm{J}=6.3 \mathrm{~Hz}), 1.00(\mathrm{t}$, $3 \mathrm{H}, \mathrm{J}=7.3 \mathrm{~Hz}$ ), $1.13(\mathrm{~d}, 9 \mathrm{H}, \mathrm{J}=7.3 \mathrm{~Hz}), 1.14(\mathrm{~d}, 9 \mathrm{H}, \mathrm{J}=7.3 \mathrm{~Hz}$ ), 1.28 (sept., 3H, J = $7.4 \mathrm{~Hz}), 1.58-1.72(\mathrm{~m}, 2 \mathrm{H}), 2.12-2.40(\mathrm{~m}, 3 \mathrm{H}), 2.41-2.57(\mathrm{~m}, 2 \mathrm{H}), 2.70-2.81(\mathrm{~m}, 1 \mathrm{H})$, 
3.15 (sept., $1 \mathrm{H}, \mathrm{J}=6.2 \mathrm{~Hz}), 3.72(\mathrm{~s}, 3 \mathrm{H}), 3.81(\mathrm{~s}, 3 \mathrm{H}), 3.87(\mathrm{~s}, 6 \mathrm{H}), 5.15(\mathrm{~d}, 1 \mathrm{H}, \mathrm{J}=5.3$ $\mathrm{Hz}), 6.53(\mathrm{~s}, 1 \mathrm{H}), 6.70(\mathrm{~s}, 1 \mathrm{H}) ;{ }^{13} \mathrm{C}-\mathrm{NMR}\left(75 \mathrm{MHz}, \mathrm{CDCl}_{3}\right) \delta 14.1,14.5,18.3,21.1$, $22.7,23.9,30.7,33.6,38.9,55.8,60.3,60.7,66.3,68.9,106.3,111.2,122.7,123.6,130.1$, $131.5,135.7,140.0,145.6,151.0,151.4,152.0$, based on integration, signals at 18.3 and 55.8 ppm may each contain $2\left(\mathrm{CH}_{3}\right)$; IR (mix. of diastereomers) $\left(\mathrm{KBr}, \mathrm{cm}^{-1}\right) 2964(\mathrm{~s})$, $1597(\mathrm{~m}), 1463$ (s), 1236 (s), 1104 (s); mass spectrum (EI) $\mathrm{m} / \mathrm{z}$ (\% rel. intensity) $586 \mathrm{M}^{+}$ (100), 501 (17), 485 (21), $483 \mathrm{M}^{+}$- $i$-PrOH - i-Pr (14), 470 (13), 370 (12), 256 (15), 236 (37), 181 (23), 129 (39), 97 (62). Anal (mix of diastereomers) calcd for $\mathrm{C}_{34} \mathrm{H}_{54} \mathrm{O}_{6} \mathrm{Si}$ : C, 69.58; H, 9.27. Found C, 69.64; H, 9.27.

Protected phenol 12d: $\mathrm{R}=\mathrm{Me}, \mathrm{R}^{1}=t-\mathrm{Bu}\left(\mathrm{a} S^{*}, 7 S^{*}\right)$ diastereomer 12d,II: Yield 85 $\%$. Colorless crystals. M.p. $124.0-125.0^{\circ} \mathrm{C}$.

${ }^{1} \mathrm{H}-\mathrm{NMR}\left(500 \mathrm{MHz}, \mathrm{CDCl}_{3}\right) \delta 0.92(\mathrm{t}, 3 \mathrm{H}, \mathrm{J}=7.4 \mathrm{~Hz}), 0.97(\mathrm{~s}, 9 \mathrm{H}), 1.13(\mathrm{~d}, 9 \mathrm{H}, \mathrm{J}=7.5$ Hz), 1.14 (d, 9H, J = 7.5 Hz), 1.38 (sept., 3H, J = 7.5 Hz), 1.50-1.64 (m, 2H), 1.91-2.00 (m, 1H), 2.22-2.43 (m, 4H), $2.91(\mathrm{ddd}, 1 \mathrm{H}, \mathrm{J}=13.4,9.2,6.3 \mathrm{~Hz}), 3.65(\mathrm{~s}, 3 \mathrm{H}), 3.68(\mathrm{~s}$, 3H), $3.90(\mathrm{~s}, 3 \mathrm{H}), 3.91(\mathrm{~s}, 3 \mathrm{H}), 4.24(\mathrm{dd}, 1 \mathrm{H}, \mathrm{J}=11.8,6.7 \mathrm{~Hz}), 6.55(\mathrm{~s}, 1 \mathrm{H}), 6.62(\mathrm{~s}, 1 \mathrm{H})$; ${ }^{13} \mathrm{C}-\mathrm{NMR}\left(125 \mathrm{MHz}, \mathrm{CDCl}_{3}\right) \delta 13.8,14.4,18.4,18.6,24.6,28.1,31.3,34.2,39.9,55.9$, $56.3,60.87,60.90,72.2,74.1,106.9,111.1,121.3,121.6,132.4,133.5,135.9,140.4$ 146.4, 150.1, 151.8, 152.5; IR (mix. of diastereomers) $\left(\mathrm{KBr}, \mathrm{cm}^{-1}\right) 2945(\mathrm{~s}), 1597(\mathrm{~m})$, 1465 (s), 1229 (s); mass spectrum (EI) $m / z$ (\% rel. intensity) $600 \mathrm{M}^{+}(100), 543 \mathrm{M}^{+}-t$ $\mathrm{Bu}$ (12), 501 (62), 469 (14), 73 (14), 57 (33); HRMS (EI) calcd for $\mathrm{C}_{35} \mathrm{H}_{56} \mathrm{O}_{6} \mathrm{Si} 600.3846$, found 600.3852 .

$\left(\mathrm{a} R^{*}, 7 S^{*}\right)$ diastereomer 12d,I: Colorless crystals. M.p. $111.5-112.5^{\circ} \mathrm{C}$. 
${ }^{1} \mathrm{H}-\mathrm{NMR}\left(500 \mathrm{MHz}, \mathrm{CDCl}_{3}\right) \delta 0.86(\mathrm{~s}, 9 \mathrm{H}), 0.93(\mathrm{t}, 3 \mathrm{H}, \mathrm{J}=7.4 \mathrm{~Hz}), 1.14(\mathrm{~d}, 9 \mathrm{H}, \mathrm{J}=7.5$ Hz), $1.15(\mathrm{~d}, 9 \mathrm{H}, \mathrm{J}=7.5 \mathrm{~Hz}), 1.30$ (sept., 3H, J = 7.5 Hz), 1.53-1.63 (m, 2H), 2.08 (td, $1 \mathrm{H}, \mathrm{J}=13.7,5.4 \mathrm{~Hz}), 2.28-2.43(\mathrm{~m}, 3 \mathrm{H}), 2.49(\mathrm{td}, 1 \mathrm{H}, \mathrm{J}=13.1,6.2 \mathrm{~Hz}), 2.75-2.84(\mathrm{~m}$, 1H), $3.72(\mathrm{~s}, 3 \mathrm{H}), 3.74(\mathrm{~s}, 3 \mathrm{H}), 3.82(\mathrm{~s}, 3 \mathrm{H}), 3.88(\mathrm{~s}, 3 \mathrm{H}), 5.06(\mathrm{~d}, 1 \mathrm{H}, \mathrm{J}=6.2 \mathrm{~Hz}), 6.53$ (s, 1H), $6.64(\mathrm{~s}, 1 \mathrm{H}) ;{ }^{13} \mathrm{C}-\mathrm{NMR}\left(75 \mathrm{MHz}, \mathrm{CDCl}_{3}\right) \delta 13.8,14.6,18.2,18.3,24.3,27.8$, $30.8,33.7,42.4,55.8,55.9,60.5,60.6,65.0,73.3,106.3,110.9,123.7,131.3,133.8$, 136.0, 140.0, 143.9, 151.2, 151.5, 151.7, one (C) could not be located; IR (mix. of diastereomers) $\left(\mathrm{KBr}, \mathrm{cm}^{-1}\right) 2945(\mathrm{~s}), 1597(\mathrm{~m}), 1465(\mathrm{~m}), 1229(\mathrm{~s})$; mass spectrum (EI) $m / z$ (\% rel. intensity) $600 \mathrm{M}^{+}$(100), $543 \mathrm{M}^{+}$- $t$-Bu (10), 515 (8), 501 (58), 469 (7), 370 (5), 73 (9), 59 (14); HRMS (EI) calcd for $\mathrm{C}_{35} \mathrm{H}_{56} \mathrm{O}_{6} \mathrm{Si} 600.3846$, found 600.3853.

Protected phenol 12e: $\mathrm{R}=\mathrm{Et}, \mathrm{R}^{1}=\mathrm{Me}\left(\mathrm{a} S^{*}, 7 S^{*}\right)$ diastereommer 12e,II: Yield 86 $\%$. Colorless crystals. M.p. $159.4-160.5^{\circ} \mathrm{C}$.

${ }^{1} \mathrm{H}-\mathrm{NMR}\left(600 \mathrm{MHz}, \mathrm{CDCl}_{3}\right) \delta 0.95(\mathrm{t}, 3 \mathrm{H}, \mathrm{J}=7.4 \mathrm{~Hz}), 1.12(\mathrm{~d}, 9 \mathrm{H}, \mathrm{J}=7.5 \mathrm{~Hz}), 1.13(\mathrm{~d}$, 9H, J = 7.5 Hz), 1.19 (t, 3H, J = 7.0 Hz), 1.39 (sept., 3H, J = 7.6 Hz), 1.54-1.67 (m, 2H), 2.18-2.27 (m, 2H), 2.33-2.43 (m, 3H), 2.88 (ddd, 1H, J = 13.6, 9.5, 6.1 Hz), $3.23(\mathrm{~s}, 3 \mathrm{H})$, 3.49 (s, 3H), 3.84-3.89 (m, 2H), 3.90 (s, 3H), 3.91 (s, 3H), $4.01(\mathrm{dd}, 1 \mathrm{H}, \mathrm{J}=10.2,8.3$ $\mathrm{Hz}), 6.54(\mathrm{~s}, 1 \mathrm{H}), 6.73(\mathrm{~s}, 1 \mathrm{H}) ;{ }^{13} \mathrm{C}-\mathrm{NMR}\left(75 \mathrm{MHz}, \mathrm{CDCl}_{3}\right) \delta 13.8,14.6,15.0,18.3$, $18.4,24.2,30.7,33.4,37.9,55.9,57.6,60.3,61.0,65.3,82.2,106.7,114.2,121.8,123.3$, $127.4,133.9,135.3,140.5,145.2,150.0,151.8,152.5$; IR (mix. of diastereomers) $(\mathrm{KBr}$, $\left.\mathrm{cm}^{-1}\right) 2935(\mathrm{~s}), 1598(\mathrm{~m}), 1466(\mathrm{~s}), 1227(\mathrm{~s}), 1103(\mathrm{~s})$; mass spectrum (EI) $\mathrm{m} / \mathrm{z}$ (\% rel. intensity) $572 \mathrm{M}^{+}(85), 540 \mathrm{M}^{+}-\mathrm{MeOH}(17), 529 \mathrm{M}^{+}-i-\operatorname{Pr}(32), 497 \mathrm{M}^{+}-\mathrm{MeOH}-i-\mathrm{Pr}$ (100), $482 \mathrm{M}^{+}-\mathrm{MeOH}-i-\mathrm{Pr}-\mathrm{Me}(32), 468(19), 384 \mathrm{M}^{+}-\mathrm{MeO}-\mathrm{Si}(i-\operatorname{Pr})_{3}(15), 89$ 
(12), 75 (10). Anal (mix of diastereomers) calcd for $\mathrm{C}_{33} \mathrm{H}_{52} \mathrm{O}_{6} \mathrm{Si}$ : C, 69.19; H, 9.15. Found C, 69.12; H, 9.04.

$\left(\mathrm{a} R^{*}, 7 S^{*}\right)$ diastereomer 12e,I: Colorless crystals. M.p. $144.8-146.2{ }^{\circ} \mathrm{C}$.

${ }^{1} \mathrm{H}-\mathrm{NMR}\left(600 \mathrm{MHz}, \mathrm{CDCl}_{3}\right) \delta 0.98(\mathrm{t}, 3 \mathrm{H}, \mathrm{J}=7.3 \mathrm{~Hz}), 1.14(\mathrm{~d}, 9 \mathrm{H}, \mathrm{J}=7.5 \mathrm{~Hz}), 1.15(\mathrm{~d}$, 9H, J = 7.5 Hz), 1.20 (t, 3H, J = 6.9 Hz), 1.29 (sept., 3H, J = 7.5 Hz), 1.59-1.70 (m, 2H), $2.24(\mathrm{td}, 1 \mathrm{H}, \mathrm{J}=14.0,5.3 \mathrm{~Hz}), 2.31-2.38(\mathrm{~m}, 2 \mathrm{H}), 2.44-2.53(\mathrm{~m}, 2 \mathrm{H}), 2.76(\mathrm{ddd}, 1 \mathrm{H}, \mathrm{J}=$ 14.0, 9.4, $6.7 \mathrm{~Hz}), 2.90$ (s, 3H), 3.59 (s, 3H), 3.83-3.91 (m, 2H), 3.869 (s, 3H), 3.873 (s, $3 \mathrm{H}), 4.91(\mathrm{~d}, 1 \mathrm{H}, \mathrm{J}=6.6 \mathrm{~Hz}), 6.51(\mathrm{~s}, 1 \mathrm{H}), 6.77(\mathrm{~s}, 1 \mathrm{H}) ;{ }^{13} \mathrm{C}-\mathrm{NMR}\left(75 \mathrm{MHz}, \mathrm{CDCl}_{3}\right) \delta$ $13.9,14.4,15.0,18.20,18.23,23.7,30.8,33.2,39.1,55.7,60.5,60.7,65.5,74.3,106.0$, 115.1, 123.2, 124.6, 129.1, 131.6, 135.1, 140.3, 146.7, 151.0, 151.9, 152.1, based on integration, signal at $55.7 \mathrm{ppm}$ may contain $2\left(\mathrm{CH}_{3}\right)$; IR (mix. of diastereomers) $(\mathrm{KBr}$, $\left.\mathrm{cm}^{-1}\right) 2935(\mathrm{~s}), 1598(\mathrm{~m}), 1466(\mathrm{~s}), 1227(\mathrm{~s}), 1103(\mathrm{~s})$; mass spectrum (EI) $\mathrm{m} / \mathrm{z}$ (\% rel. intensity) $572 \mathrm{M}^{+}(100), 529 \mathrm{M}^{+}-i-\operatorname{Pr}(24), 497 \mathrm{M}^{+}-\mathrm{MeOH}-i-\operatorname{Pr}(89), 482 \mathrm{M}^{+}-\mathrm{MeOH}$ - $i$-Pr $-\mathrm{Me}$ (32), 468 (15), $384 \mathrm{M}^{+}-\mathrm{MeO}-\mathrm{Si}(i-\operatorname{Pr})_{3}(14), 236$ (30), 159 (31), 91 (46). Anal (mix of diastereomers) calcd for $\mathrm{C}_{33} \mathrm{H}_{52} \mathrm{O}_{6} \mathrm{Si}$ : C, 69.19; H, 9.15. Found C, 69.12; H, 9.04 .

Protected phenol 12f: $\mathrm{R}=i-\operatorname{Pr}, \mathrm{R}^{1}=\operatorname{Me}\left(\mathrm{a} S^{*}, 7 S^{*}\right)$ diastereomer 12f,II: Yield 91 $\%$. Colorless crystals. M.p. $162.3-163.1^{\circ} \mathrm{C}$.

${ }^{1} \mathrm{H}-\mathrm{NMR}\left(600 \mathrm{MHz}, \mathrm{CDCl}_{3}\right) \delta 0.85(\mathrm{~d}, 3 \mathrm{H}, \mathrm{J}=6.2 \mathrm{~Hz}), 0.93(\mathrm{t}, 3 \mathrm{H}, \mathrm{J}=7.3 \mathrm{~Hz}), 1.11-$ $1.15(\mathrm{~m}, 21 \mathrm{H}), 1.39$ (sept., 3H, J = 7.5 Hz), 1.54-1.65 (m, 2H), 2.18-2.27 (m, 2H), 2.302.44 (m, 3H), 2.87 (ddd, 1H, J = 13.6, 9.0, 6.6 Hz), 3.24 (s, 3H), 3.51 (s, 3H), 3.84 (sept., 1H, J = 6.1 Hz), $3.90(\mathrm{~s}, 3 \mathrm{H}), 3.91(\mathrm{~s}, 3 \mathrm{H}), 4.05(\mathrm{dd}, 1 \mathrm{H}, \mathrm{J}=10.5,7.8 \mathrm{~Hz}), 6.53(\mathrm{~s}, 1 \mathrm{H})$, $6.76(\mathrm{~s}, 1 \mathrm{H}) ;{ }^{13} \mathrm{C}-\mathrm{NMR}\left(125 \mathrm{MHz}, \mathrm{CDCl}_{3}\right) \delta 13.7,14.6,18.3,18.4,21.8,22.1,24.2$, 
$30.7,33.3,37.9,56.0,57.6,60.4,61.1,73.1,82.2,106.5,119.0,122.1,125.5,127.3$, 134.0, 135.0, 140.6, 146.0, 148.8, 151.8, 152.5; IR (mix. of diastereomers) $\left(\mathrm{KBr}, \mathrm{cm}^{-1}\right)$ $2940(\mathrm{~s}), 1598(\mathrm{~m}), 1462(\mathrm{~s}), 1224(\mathrm{~s}), 1129(\mathrm{~s})$; mass spectrum (EI) m/z (\% rel. intensity) $586 \mathrm{M}^{+}(100), 554 \mathrm{M}^{+}-\mathrm{MeOH}(16), 515 \mathrm{M}^{+}-i$-Pr (17), $511 \mathrm{M}^{+}-\mathrm{MeOH}-i$-Pr (55), 501 (32), 469 (100), 454 (20), 356 (20), 131 (13), 117 (19), 84 (46). Anal (mix of diastereomers) calcd for $\mathrm{C}_{34} \mathrm{H}_{54} \mathrm{O}_{6} \mathrm{Si}: \mathrm{C}, 69.58 ; \mathrm{H}, 9.27$. Found C, 69.61; H, 9.29. $\left(\mathrm{a} R^{*}, 7 S^{*}\right)$ diastereomer 12f,I: Colorless crystals. M.p. $145.6-146.4{ }^{\circ} \mathrm{C}$. ${ }^{1} \mathrm{H}-\mathrm{NMR}\left(600 \mathrm{MHz}, \mathrm{CDCl}_{3}\right) \delta 0.87(\mathrm{~d}, 3 \mathrm{H}, \mathrm{J}=5.9 \mathrm{~Hz}), 0.96(\mathrm{t}, 3 \mathrm{H}, \mathrm{J}=7.3 \mathrm{~Hz}),, 1.11-$ $1.17(\mathrm{~m}, 21 \mathrm{H}), 1.30$ (sept., 3H, J = 7.5 Hz), 1.60-1.67 (m, 2H), 2.25 (td, 1H, J = 14.1, 5.3 $\mathrm{Hz}), 2.32-2.38(\mathrm{~m}, 2 \mathrm{H}), 2.42-2.50(\mathrm{~m}, 2 \mathrm{H}), 2.72-2.79(\mathrm{~m}, 1 \mathrm{H}), 2.91(\mathrm{~s}, 3 \mathrm{H}), 3.60(\mathrm{~s}, 3 \mathrm{H})$, 3.84 (sept., 1H, J = 6.1 Hz), 3.876 (s, 3H), 3.884 (s, 3H), 4.91 (d, 1H, J = 6.6 Hz), 6.50 (s, $1 \mathrm{H}), 6.81(\mathrm{~s}, 1 \mathrm{H}) ;{ }^{13} \mathrm{C}-\mathrm{NMR}\left(75 \mathrm{MHz}, \mathrm{CDCl}_{3}\right) \delta 13.8,14.4,18.18,18.20,21.9,22.2$, 23.6, 30.8, 33.0, 39.1, 55.66, 55.71, 60.4, 60.7, 73.3, 74.3, 105.8, 120.0, 123.6, 126.9, 129.1, 131.7, 134.9, 140.4, 147.5, 149.8, 151.8, 152.1; IR (mix. of diastereomers) $(\mathrm{KBr}$, $\left.\mathrm{cm}^{-1}\right) 2940(\mathrm{~s}), 1598(\mathrm{~m}), 1462(\mathrm{~s}), 1224(\mathrm{~s}), 1129(\mathrm{~s})$; mass spectrum (EI) $\mathrm{m} / \mathrm{z}$ (\% rel. intensity) $586 \mathrm{M}^{+}(100), 515 \mathrm{M}^{+}-i-\operatorname{Pr}(11), 511 \mathrm{M}^{+}-\mathrm{MeOH}-i-\operatorname{Pr}(34), 501$ (23), 469 (79), 454 (17), 356 (18), 326 (22), 292 (12), 204 (10), 165 (13), 89 (15). Anal (mix of diastereomers) calcd for $\mathrm{C}_{34} \mathrm{H}_{54} \mathrm{O}_{6} \mathrm{Si}$ C, 69.58; H, 9.27. Found C, 69.61; H, 9.29. 
$\left(\mathrm{aR}^{*}, 7 \mathrm{~S}^{*}\right)$-7,11-dialkoxy-8-hydroxy-1,2,3-trimethoxy-9-propyl-5,6-dihydro-5Hdibenzo[a,c]cycloheptenes (2,I). General Procedure.

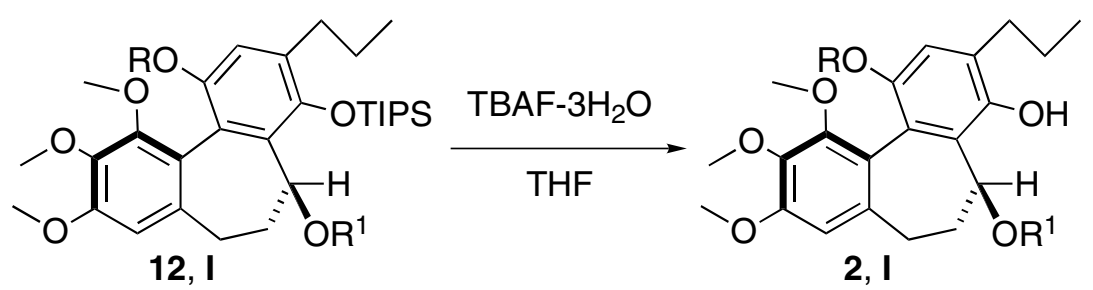

To a solution of the proper TIPS - protected (a $\left.R^{*}, 7 S^{*}\right)$ phenol 12,I ( 1 eq.) in THF $(\sim 0.05 \mathrm{M})$ was added TBAF- $3 \mathrm{H}_{2} \mathrm{O}(1.25$ eq. $)$ in one portion. The mixture was stirred for 0.5 hours and monitored by TLC for the disappearance of the starting material. After the reaction was complete, the solvent was removed in vacuo, the residue was purified on a short column (silica gel, $25 \%$ EtOAc in hexane). The products and yields are given below:

Phenol 2a,I: R, $\mathrm{R}^{1}=$ Me. Yield $93 \%$. Colorless crystals. M.p. $158.5-159.5^{\circ} \mathrm{C}$. ${ }^{1} \mathrm{H}-\mathrm{NMR}\left(500 \mathrm{MHz}\right.$, Acetone-d $\left.\mathrm{d}_{6}\right) \delta 0.99(\mathrm{t}, 3 \mathrm{H}, \mathrm{J}=7.3 \mathrm{~Hz}), 1.64-1.73(\mathrm{~m}, 2 \mathrm{H}), 2.11-2.23$ (m, 2H), 2.29-2.35 (m, 2H), $2.69(\mathrm{dd}, 2 \mathrm{H}, \mathrm{J}=9.1,6.6 \mathrm{~Hz}), 2.81(\mathrm{~s}, 3 \mathrm{H}), 3.67(\mathrm{~s}, 3 \mathrm{H}), 3.70$ (s, 3H), $3.75(\mathrm{~s}, 3 \mathrm{H}), 3.81(\mathrm{~s}, 3 \mathrm{H}), 5.11(\mathrm{dd}, 1 \mathrm{H}, \mathrm{J}=6.0,1.4 \mathrm{~Hz}), 6.55(\mathrm{~s}, 1 \mathrm{H}), 6.77(\mathrm{~s}$, 1H, OH), $6.80(\mathrm{~s}, 1 \mathrm{H}) ;{ }^{1} \mathrm{H}-\mathrm{NMR}\left(500 \mathrm{MHz}, \mathrm{CDCl}_{3}\right) \delta 1.05(\mathrm{t}, 3 \mathrm{H}, \mathrm{J}=7.4 \mathrm{~Hz}), 1.65-1.78$ (m, 2H), 2.18-2.40 (m, 3H), 2.42-2.53 (m, 1H), 2.53-2.67 (m, 2H), $2.92(\mathrm{~s}, 3 \mathrm{H}), 3.72(\mathrm{~s}$, 3H), 3.78 (s, 3H), 3.85 (s, 3H), 3.86 (s, 3H), 4.42 (s, 1H, OH), 5.05-5.11 (br.s, 1H), 6.53 (s, 1H), 6.71-6.77 (br.s, 1H); ${ }^{13} \mathrm{C}-\mathrm{NMR}\left(125 \mathrm{MHz}\right.$, Acetone-d $\left.{ }_{6}\right) \delta$ 14.3, 24.0, 31.2, 33.5, $40.0,55.6,56.1,56.3,60.5,60.7,74.6,107.4,113.2,123.9,124.8,128.0,129.7,136.5$, 141.3, 146.6, 151.8, 152.7, 153.1; IR (KBr, cm $\left.{ }^{-1}\right) 3458(\mathrm{~s}, \mathrm{OH}), 3215$ (s, OH), 2934 (s), 1600 (m), 1477 (s), 1225 (s); mass spectrum (EI) $\mathrm{m} / z$ (\% rel. intensity) $402 \mathrm{M}^{+}$(31), 370 $\mathrm{M}^{+}$- MeOH (100), 355 (12), 339 (20), 324 (11), 295 (5), 185 (10); HRMS (EI) calcd for 
$\mathrm{C}_{23} \mathrm{H}_{30} \mathrm{O}_{6}$ 402.2042, found 402.2056. Anal calcd for $\mathrm{C}_{23} \mathrm{H}_{30} \mathrm{O}_{6}$ : C, 68.64; H, 7.51. Found C, 68.49; H, 7.78.

Phenol 2b,I: $\mathrm{R}=\mathrm{Me}, \mathrm{R}^{1}=$ Et. Yield $91 \%$. Colorless crystals. M.p. $161.6-162.3$ ${ }^{\circ} \mathrm{C}$.

${ }^{1} \mathrm{H}-\mathrm{NMR}\left(600 \mathrm{MHz}, \mathrm{CDCl}_{3}\right) \delta 0.84(\mathrm{t}, 3 \mathrm{H}, \mathrm{J}=7.0 \mathrm{~Hz}), 1.06(\mathrm{t}, 3 \mathrm{H}, \mathrm{J}=7.4 \mathrm{~Hz}), 1.67-$ $1.77(\mathrm{~m}, 2 \mathrm{H}), 2.22-2.29(\mathrm{~m}, 2 \mathrm{H}), 2.35-2.40(\mathrm{~m}, 1 \mathrm{H}), 2.45-2.53(\mathrm{~m}, 1 \mathrm{H}), 2.56-2.62(\mathrm{~m}$, 2H), 3.02-3.13 (m, 2H), $3.73(\mathrm{~s}, 3 \mathrm{H}), 3.82(\mathrm{~s}, 3 \mathrm{H}), 3.87(\mathrm{~s}, 3 \mathrm{H}), 3.88(\mathrm{~s}, 3 \mathrm{H}), 4.32(\mathrm{~s}, 1 \mathrm{H}$, OH), 5.15-5.19 (br.s, 1H), 6.55 (s, 1H), 6.72 (br.s, 1H); ${ }^{13} \mathrm{C}-\mathrm{NMR}$ (75 MHz, Acetone-d $\mathrm{d}_{6}$ ) $\delta 14.3,15.3,24.0,31.1,33.5,39.7,55.07,55.12,60.3,60.5,62.9,72.5,107.4,112.7$, 123.9, 124.8, 128.6, 129.5, 136.6, 141.3, 146.2, 151.6, 152.4, 152.9; IR $\left(\mathrm{KBr}, \mathrm{cm}^{-1}\right) 3474$ (s, OH), $2934(\mathrm{~s}), 1599(\mathrm{~m}), 1463(\mathrm{~m}), 1223(\mathrm{~m}), 1119(\mathrm{~s})$; mass spectrum (EI) m/z (\% rel. intensity) $416 \mathrm{M}^{+}$(2), $370 \mathrm{M}^{+}$- EtOH (40), 314 (8), 236 (91), 206 (47), 178 (15), 129 (66), 91 (100). Anal calcd for $\mathrm{C}_{24} \mathrm{H}_{32} \mathrm{O}_{6}$ : C, 69.21; H, 7.74. Found C, 69.01; H, 7.64.

Phenol 2c,I: $\mathrm{R}=\mathrm{Me}, \mathrm{R}^{1}=i$-Pr. Yield $92 \%$. Colorless crystals. M.p. $166.4-$ $167.1{ }^{\circ} \mathrm{C}$.

${ }^{1} \mathrm{H}-\mathrm{NMR}\left(600 \mathrm{MHz}, \mathrm{CDCl}_{3}\right) \delta 0.73(\mathrm{~d}, 3 \mathrm{H}, \mathrm{J}=6.1 \mathrm{~Hz}), 0.97(\mathrm{~d}, 3 \mathrm{H}, \mathrm{J}=6.1 \mathrm{~Hz}), 1.07(\mathrm{t}$, $3 \mathrm{H}, \mathrm{J}=7.4 \mathrm{~Hz}), 1.68-1.78(\mathrm{~m}, 2 \mathrm{H}), 2.16-2.29(\mathrm{~m}, 2 \mathrm{H}), 2.38(\mathrm{dd}, 1 \mathrm{H}, \mathrm{J}=13.2,5.6 \mathrm{~Hz})$, 2.45-2.52 (m, 1H), 2.55-2.62 (m, 2H), 3.21 (sept., 1H, J = 6.1 Hz), 3.73 (br.s, 3H), 3.80 (s, 3H), 3.87 (s, 3H), 3.96 (s, 3H), 4.34 (s, 1H, OH), 5.30-5.38 (br.s, 1H), 6.56 (s, 1H), 6.71 (br.s, $1 \mathrm{H}) ;{ }^{13} \mathrm{C}-\mathrm{NMR}\left(75 \mathrm{MHz}, \mathrm{CDCl}_{3}\right) \delta 14.2,20.3,22.8,22.9,30.6,32.8,39.1$, $55.7,55.9,60.1,60.7,66.4,67.9,106.4,111.3,122.1,124.3,126.6,126.9,136.0,139.8$, 144.7, 150.6, 151.3, 152.2; IR (KBr, cm-1) 3532 (s, OH), 2933 (s), 1599 (m), 1462 (m), 1124 (s); mass spectrum (EI) $m / z$ (\% rel. intensity) $430 \mathrm{M}^{+}$(7), $370 \mathrm{M}^{+}$- $i$-PrOH (100), 
355 (8), 339 (14), 324 (7). Anal calcd for $\mathrm{C}_{25} \mathrm{H}_{34} \mathrm{O}_{6}$ : C, 69.74; H, 7.96. Found C, 69.57; H, 7.84 .

Phenol 2d,I: $\mathrm{R}=\mathrm{Me}, \mathrm{R}^{1}=t$-Bu. Yield $96 \%$. Colorlesss crystals. M.p. 135.5$136.5^{\circ} \mathrm{C}$.

${ }^{1} \mathrm{H}-\mathrm{NMR}\left(500 \mathrm{MHz}, \mathrm{CDCl}_{3}\right) \delta 0.90(\mathrm{~s}, 9 \mathrm{H}), 1.04(\mathrm{t}, 3 \mathrm{H}, \mathrm{J}=7.4 \mathrm{~Hz}), 1.65-1.76(\mathrm{~m}, 2 \mathrm{H})$, $2.10(\mathrm{td}, 1 \mathrm{H}, \mathrm{J}=13.4,6.0 \mathrm{~Hz}), 2.21-2.30(\mathrm{~m}, 1 \mathrm{H}), 2.33(\mathrm{dd}, 1 \mathrm{H}, \mathrm{J}=13.2,5.9 \mathrm{~Hz}), 2.43$ $(\mathrm{td}, 1 \mathrm{H}, \mathrm{J}=13.0,6.9 \mathrm{~Hz}), 2.58(\mathrm{t}, 2 \mathrm{H}, \mathrm{J}=7.7 \mathrm{~Hz}), 3.71(\mathrm{~s}, 3 \mathrm{H}), 3.82(\mathrm{~s}, 3 \mathrm{H}), 3.83(\mathrm{~s}, 3 \mathrm{H})$, $3.88(\mathrm{~s}, 3 \mathrm{H}), 4.36(\mathrm{~s}, 1 \mathrm{H}, \mathrm{OH}), 5.24(\mathrm{~d}, 1 \mathrm{H}, \mathrm{J}=5.6 \mathrm{~Hz}), 6.54(\mathrm{~s}, 1 \mathrm{H}), 6.66(\mathrm{~s}, 1 \mathrm{H}) ;{ }^{13} \mathrm{C}-$ NMR $\left(125 \mathrm{MHz}, \mathrm{CDCl}_{3}\right) \delta 14.0,22.9,28.0,30.6,32.7,41.8,55.9,56.2,60.48,60.54$, 64.2, 73.1, 106.6, 111.1, 123.4, 124.7, 126.2, 130.9, 136.4, 140.1, 142.7, 150.9, 151.4, 151.9; IR (KBr, cm $\left.{ }^{-1}\right) 3531(\mathrm{~s}, \mathrm{OH}), 2933$ (s), $1599(\mathrm{~m}), 1462$ (s), $1196(\mathrm{~m})$; mass spectrum (EI) $m / z$ (\% rel. intensity) $444 \mathrm{M}^{+}$(7), $370 \mathrm{M}^{+}-t$-BuOH (100), 355 (9), 339 (14), 324 (7), 295 (4); HRMS (EI) calcd for $\mathrm{C}_{26} \mathrm{H}_{36} \mathrm{O}_{6} 444.2512$, found 444.2517.

Phenol 2e, $\mathbf{I}^{1}: \mathbf{R}=\mathrm{Et}, \mathrm{R}^{1}=$ Me. Yield $92 \%$. Colorless crystals. M.p. $148.8-150.1$ ${ }^{\circ} \mathrm{C}$.

${ }^{1} \mathrm{H}-\mathrm{NMR}\left(300 \mathrm{MHz}, \mathrm{CDCl}_{3}\right) \delta 1.05(\mathrm{t}, 3 \mathrm{H}, \mathrm{J}=7.5 \mathrm{~Hz}), 1.21(\mathrm{t}, 3 \mathrm{H}, \mathrm{J}=6.9 \mathrm{~Hz}), 1.66-1.76$ (m, 2H), 2.25-2.40 (m, 3H), $2.45(\mathrm{dd}, 1 \mathrm{H}, \mathrm{J}=13.2,6.6 \mathrm{~Hz}), 2.55-2.61(\mathrm{~m}, 2 \mathrm{H}),, 2.95(\mathrm{~s}$, $3 \mathrm{H}), 3.71(\mathrm{~s}, 3 \mathrm{H}), 3.86(\mathrm{~m}, 2 \mathrm{H}), 3.86(\mathrm{~s}, 3 \mathrm{H}), 3.87(\mathrm{~s}, 3 \mathrm{H}), 4.33(\mathrm{~s}, 1 \mathrm{H}, \mathrm{OH}$, exchange with $\left.\mathrm{D}_{2} \mathrm{O}\right), 5.07(\mathrm{~d}, 1 \mathrm{H}, \mathrm{J}=5.1 \mathrm{~Hz}), 6.53(\mathrm{~s}, 1 \mathrm{H}), 6.77(\mathrm{~s}, 1 \mathrm{H}) ;{ }^{13} \mathrm{C}-\mathrm{NMR}(75 \mathrm{MHz}$, $\left.\mathrm{CDCl}_{3}\right) \delta 14.1,15.1,22.7,30.7,32.6,39.4,55.6,55.9,60.6,60.7,65.8,73.8,106.1$, 115.5, 122.6, 125.4, 125.7, 126.6, 135.4, 140.2, 145.5, 150.7, 151.9, 152.0; IR $\left(\mathrm{KBr}, \mathrm{cm}^{-}\right.$ 1) $3440(\mathrm{~s}, \mathrm{OH}), 2936(\mathrm{~s}), 1601(\mathrm{~m}), 1466(\mathrm{~s}), 1231(\mathrm{~m}), 1105(\mathrm{~m})$; mass spectrum (EI) $m / z$ (\% rel. intensity) $416 \mathrm{M}^{+}$(38), $384 \mathrm{M}^{+}-\mathrm{MeOH}$ (100), 355 (21), 324 (25), 295 (10); 
HRMS (EI) calcd for $\mathrm{C}_{24} \mathrm{H}_{32} \mathrm{O}_{6} 416.2199$, found 416.2218. Anal (mix of diastereomers) calcd for $\mathrm{C}_{24} \mathrm{H}_{32} \mathrm{O}_{6}$ : C, 69.21; H, 7.74. Found C, 69.05; H, 7.71.

Phenol 2f, $\mathbf{I}^{1}: \mathrm{R}=i$-Pr, $\mathrm{R}^{1}=$ Me. Yield $91 \%$. Colorless crystals. M.p. $157.9-$ $158.9^{\circ} \mathrm{C}$.

${ }^{1} \mathrm{H}-\mathrm{NMR}\left(500 \mathrm{MHz}, \mathrm{C}_{6} \mathrm{D}_{6}\right) \delta 0.84(\mathrm{t}, 3 \mathrm{H}, \mathrm{J}=7.4 \mathrm{~Hz}), 0.95(\mathrm{~d}, 3 \mathrm{H}, \mathrm{J}=6.1 \mathrm{~Hz}), 1.20(\mathrm{~d}$, $3 \mathrm{H}, \mathrm{J}=6.2 \mathrm{~Hz}), 1.45-1.55(\mathrm{~m}, 2 \mathrm{H}), 2.19-2.31(\mathrm{~m}, 4 \mathrm{H}), 2.44(\mathrm{dt}, 1 \mathrm{H}, \mathrm{J}=13.6,5.4 \mathrm{~Hz})$, $2.57(\mathrm{dt}, 1 \mathrm{H}, \mathrm{J}=12.9,6.2 \mathrm{~Hz}), 3.00(\mathrm{~s}, 3 \mathrm{H}), 3.41(\mathrm{~s}, 3 \mathrm{H}), 3.89(\mathrm{~s}, 3 \mathrm{H}), 3.92(\mathrm{~s}, 3 \mathrm{H}), 4.06$ (sept., $1 \mathrm{H}, \mathrm{J}=6.1 \mathrm{~Hz}$ ), 4.06-4.11 (br.s., 1H, OH), $5.20(\mathrm{~d}, 1 \mathrm{H}, \mathrm{J}=6.2 \mathrm{~Hz}), 6.38(\mathrm{~s}, 1 \mathrm{H})$, $6.78(\mathrm{~s}, 1 \mathrm{H}) ;{ }^{1} \mathrm{H}-\mathrm{NMR}\left(300 \mathrm{MHz}, \mathrm{CDCl}_{3}\right) \delta 0.88(\mathrm{~d}, 3 \mathrm{H}, \mathrm{J}=6.1 \mathrm{~Hz}), 1.04(\mathrm{t}, 3 \mathrm{H}, \mathrm{J}=7.3$ $\mathrm{Hz}), 1.16(\mathrm{~d}, 3 \mathrm{H}, \mathrm{J}=6.1 \mathrm{~Hz}), 1.62-1.81(\mathrm{~m}, 2 \mathrm{H}), 2.18-2.51(\mathrm{~m}, 4 \mathrm{H}), 2.51-2.65(\mathrm{~m}, 2 \mathrm{H})$, $2.94(\mathrm{~s}, 3 \mathrm{H}), 3.71(\mathrm{~s}, 3 \mathrm{H}), 3.84-3.91(\mathrm{~m}, 1 \mathrm{H}), 3.87(\mathrm{~s}, 6 \mathrm{H}), 4.46(\mathrm{~s}, 1 \mathrm{H}, \mathrm{OH}), 5.07(\mathrm{~d}, 1 \mathrm{H}$, $\mathrm{J}=5.5 \mathrm{~Hz}), 6.51(\mathrm{~s}, 1 \mathrm{H}), 6.75-6.83$ (br.s, $1 \mathrm{H}) ;{ }^{13} \mathrm{C}-\mathrm{NMR}\left(125 \mathrm{MHz}, \mathrm{C}_{6} \mathrm{D}_{6}\right) \delta 14.1,22.2$, $22.4,23.0,31.1,32.5,40.0,55.5,55.8,60.5,60.6,72.5,74.2,107.0,118.5,123.8,126.6$, 126.9, 127.5, 135.8, 141.4, 146.2, 150.2, 152.8, 153.0; IR $\left(\mathrm{KBr}, \mathrm{cm}^{-1}\right) 3446(\mathrm{~s}, \mathrm{OH})$, $2938(\mathrm{~s}), 1598(\mathrm{~m}), 1467(\mathrm{~s}), 1230(\mathrm{~m}), 1123(\mathrm{~m})$; mass spectrum (EI) $\mathrm{m} / \mathrm{z}$ (\% rel. intensity) $430 \mathrm{M}^{+}$(22), $398 \mathrm{M}^{+}$- MeOH (73), 356 (100), 324 (16); HRMS (EI) calcd for $\mathrm{C}_{25} \mathrm{H}_{34} \mathrm{O}_{6} 430.2355$, found 430.2357 . 
Thermal Epimerization of Phenols 2 and Their Protected Derivatives 7-12. $\mathrm{R}=\mathrm{Me}$, Et, $i$-Pr; $\mathbf{R}^{1}=\mathrm{Me}, \mathrm{Et}, i-\mathrm{Pr}, t$-Bu; $\mathbf{R}^{2}=\mathrm{H}, \mathrm{Me}, \mathrm{COCH}_{3}, \mathrm{COCMe}_{3}$, TBS, TBDMS, TIPS. General Procedure.

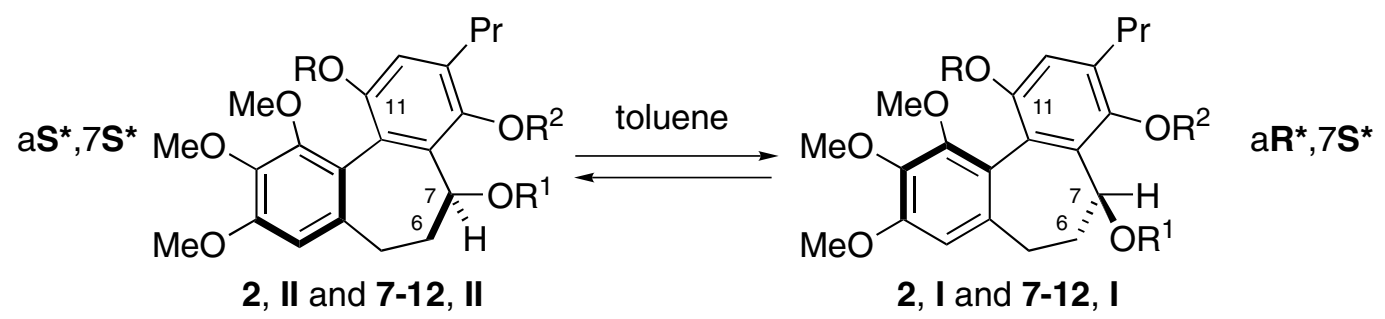

Solutions of individual diastereomers $\mathbf{I}\left(\mathrm{a} R^{*}, 7 \mathrm{~S}^{*}\right)$ and $\mathbf{I I}\left(\mathrm{a} S^{*}, 7 \mathrm{~S}^{*}\right)$ in dry toluene $(10 \mathrm{mg} / \mathrm{mL})$ were sealed in separate tubes under argon. The tubes were placed in an oil heating bath side by side, then the solutions were stirred under the described below conditions. Progress of epimerization was monitored by TLC and HPLC and the reaction was stopped as soon as the same diastereomeric ratio had been achieved in both tubes. Then the solutions were mixed together, toluene was removed in vacuo and the ratio was measured by ${ }^{1} \mathrm{H}-\mathrm{NMR}$. After that the mixture was purified chromatographically on silica gel, diastereomers I + II were collected and average material recovery was determined.

$$
\mathrm{R}, \mathrm{R}^{1}=\mathrm{Me}:
$$

7: $\mathrm{R}^{2}=\mathrm{C}(\mathrm{O}) \mathrm{Me}, 165^{\circ} \mathrm{C}, 30$ hours, $50 \%\left(\mathrm{a} R^{*}, 7 S^{*}\right), 50 \%\left(\mathrm{a} S^{*}, 7 S^{*}\right)$, recovery $(\mathbf{I}+\mathbf{I I}) 96$ $\%$.

8: $\mathrm{R}^{2}=\mathrm{C}(\mathrm{O}) t-\mathrm{Bu}, 165^{\circ} \mathrm{C}, 30$ hours, $58 \%\left(\mathrm{a} R^{*}, 7 S^{*}\right), 42 \%\left(\mathrm{a} S^{*}, 7 S^{*}\right)$, recovery $(\mathbf{I}+\mathbf{I I}) 92$ $\%$.

9: $\mathrm{R}^{2}=\mathrm{Me}, 160{ }^{\circ} \mathrm{C}, 20$ hours, $86 \%\left(\mathrm{a} R^{*}, 7 S^{*}\right), 14 \%\left(\mathrm{a} S^{*}, 7 S^{*}\right)$, recovery $(\mathbf{I}+\mathbf{I I}) 97 \%$.

10: $\mathrm{R}^{2}=\mathrm{TMS}, 160{ }^{\circ} \mathrm{C}, 20$ hours, $91 \%\left(\mathrm{a} R^{*}, 7 S^{*}\right), 9 \%\left(\mathrm{a} S^{*}, 7 S^{*}\right)$, recovery $(\mathbf{I}+$ II) $94 \%$. 11: $\mathrm{R}^{2}=$ TBDMS, $160{ }^{\circ} \mathrm{C}, 20$ hours, $95 \%\left(\mathrm{a} R^{*}, 7 S^{*}\right), 5 \%\left(\mathrm{a} S^{*}, 7 S^{*}\right)$, recovery $(\mathbf{I}+\mathbf{I I}) 99$ $\%$. 


$$
\mathrm{R}^{2}=\mathrm{TIPS}
$$

12a: $\mathrm{R}, \mathrm{R}^{1}=\mathrm{Me}, 160^{\circ} \mathrm{C}, 20$ hours, $98 \%\left(\mathrm{a} R^{*}, 7 S^{*}\right), 2 \%\left(\mathrm{a} S^{*}, 7 S^{*}\right)$, recovery $(\mathbf{I}+\mathbf{I I}) 98$ $\%$.

12b: $\mathrm{R}=\mathrm{Me}, \mathrm{R}^{1}=\mathrm{Et}, 160{ }^{\circ} \mathrm{C}, 20$ hours, $>98 \%\left(\mathrm{a} R^{*}, 7 S^{*}\right),<2 \%\left(\mathrm{a} S^{*}, 7 S^{*}\right)$, recovery $(\mathbf{I}$ + II) $97 \%$.

12c: $\mathrm{R}=\mathrm{Me}, \mathrm{R}^{1}=i$-Pr, $160{ }^{\circ} \mathrm{C}, 20$ hours, $>98 \%\left(\mathrm{a} R^{*}, 7 S^{*}\right),<2 \%\left(\mathrm{a} S^{*}, 7 S^{*}\right)$, recovery $(\mathbf{I}$ + II) $96 \%$.

12d: $\mathrm{R}=\mathrm{Me}, \mathrm{R}^{1}=t$ - $\mathrm{Bu}, 160^{\circ} \mathrm{C}, 20$ hours, $>98 \%\left(\mathrm{a} R^{*}, 7 S^{*}\right),<2 \%\left(\mathrm{a} S^{*}, 7 S^{*}\right)$, recovery

$$
\text { (I + II) } 99 \% \text {. }
$$

12e: $\mathrm{R}=\mathrm{Et}, \mathrm{R}^{1}=\mathrm{Me}, 160^{\circ} \mathrm{C}, 20$ hours, $98 \%\left(\mathrm{a} R^{*}, 7 S^{*}\right), 2 \%\left(\mathrm{a} S^{*}, 7 S^{*}\right)$, recovery $(\mathbf{I}+\mathbf{I I})$ $97 \%$.

12f: $\mathrm{R}=i-\operatorname{Pr}, \mathrm{R}^{1}=\mathrm{Me}, 160^{\circ} \mathrm{C}, 20$ hours, $98 \%\left(\mathrm{a} R^{*}, 7 S^{*}\right), 2 \%\left(\mathrm{a} S^{*}, 7 S^{*}\right)$, recovery $(\mathbf{I}+$

$$
\text { II) } 98 \% \text {. }
$$

$$
\mathrm{R}^{2}=\mathrm{H}
$$

2a: $\mathrm{R}, \mathrm{R}^{1}=\mathrm{Me}, 150{ }^{\circ} \mathrm{C}, 24$ hours, $<2 \%\left(\mathrm{a} R^{*}, 7 S^{*}\right),>98 \%\left(\mathrm{a} S^{*}, 7 S^{*}\right)$, recovery $(\mathbf{I}+\mathbf{I I})$ $68 \%$.

2a: $\mathrm{R}, \mathrm{R}^{1}=\mathrm{Me}, 150{ }^{\circ} \mathrm{C}, 28$ hours (in $i$-BuCOMe), <2 \% (aR*,7 $\left.S^{*}\right),>98 \%\left(\mathrm{a} S^{*}, 7 S^{*}\right)$,

$$
\text { recovery }(\mathbf{I}+\mathbf{I I}) 70 \% \text {. }
$$

2b: $\mathrm{R}=\mathrm{Me}, \mathrm{R}^{1}=\mathrm{Et}, 150{ }^{\circ} \mathrm{C}, 24$ hours, $<2 \%\left(\mathrm{a} R^{*}, 7 S^{*}\right),>98 \%\left(\mathrm{a} S^{*}, 7 S^{*}\right)$, recovery $(\mathbf{I}+$

$$
\text { II) } 67 \% \text {. }
$$

2c: $\mathrm{R}=\mathrm{Me}, \mathrm{R}^{1}=i$-Pr, $150{ }^{\circ} \mathrm{C}, 24$ hours, $<2 \%\left(\mathrm{a} R^{*}, 7 S^{*}\right),>98 \%\left(\mathrm{a} S^{*}, 7 S^{*}\right)$, recovery $(\mathbf{I}$

$$
\text { + II) } 68 \% \text {. }
$$


2d: $\mathrm{R}=\mathrm{Me}, \mathrm{R}^{1}=t$-Bu, $150{ }^{\circ} \mathrm{C}, 24$ hours, $<2 \%\left(\mathrm{a} R^{*}, 7 S^{*}\right),>98 \%\left(\mathrm{a} S^{*}, 7 S^{*}\right)$, recovery $(\mathbf{I}$ + II) $65 \%$.

2e: $\mathrm{R}=\mathrm{Et}, \mathrm{R}^{1}=\mathrm{Me}, 150{ }^{\circ} \mathrm{C}, 28$ hours, $<2 \%\left(\mathrm{a} R^{*}, 7 S^{*}\right),>98 \%\left(\mathrm{a} S^{*}, 7 S^{*}\right)$, recovery $(\mathbf{I}+$ II) $64 \%$.

2f: $\mathrm{R}=i$-Pr, $\mathrm{R}^{1}=\mathrm{Me}, 150{ }^{\circ} \mathrm{C}, 30$ hours, $<2 \%\left(\mathrm{a} R^{*}, 7 S^{*}\right),>98 \%\left(\mathrm{a} S^{*}, 7 S^{*}\right)$, recovery $(\mathbf{I}$ + II) $63 \%$. 